\title{
Diagnostic agro-écologique de la végétation des alpages laitiers des Alpes du Nord humides: établissement et utilisation d'une typologie simplifiée
}

\author{
A Bornard 1, M Dubost 2 \\ 1 CEMAGREF Grenoble, INERM, BP 76, 38402 Saint-Martin-d'Hères Cedex; \\ 2 International centre for alpine environments, BP 230, 73374 Le-Bourget-du-Lac Cedex, France
}

(Reçu le 5 octobre1991; accepté le 2 juin 1992)

\begin{abstract}
Résumé - La pérennité des alpages à vaches laitières des Alpes du Nord françaises dépend, notamment, d'une bonne valorisation de leurs ressources pastorales, qu'il convient de bien connaître afin de les exploiter correctement. Une typologie de la végétation de ces alpages à finalité de diagnostic agroécologique a été établie à partir de 220 relevés linéaires de végétation, répartis sur 7 alpages de référence, représentatifs de la région. Ces relevés ont été traités par des analyses multivariées: AFC, suivies de CAH. Ces traitements ont permis d'aboutir à une typologie simplifiée avec 11 types principaux de végétation et 25 sous-types. Cette typologie a plusieurs intérêts. C'est un guide de reconnaissance simplifiée des ressources pastorales, assez simple pour être utilisé par des non-spécialistes de la flore. La clé de détermination des types de végétation est basée sur l'appréciation conjointe de critères morphologiques (hauteur d'herbe), de la nature des espèces dominantes et indicatrices (25 pour la typologie simplifiée en 11 types) et des conditions de milieu et d'utilisation du secteur étudié. La classification peut être extrapolée à l'ensemble des pâturages d'altitude de la région biogéographique des Alpes externes du Nord. C'est un outil de diagnostic pastoral. Chacun des types a été caractérisé par sa valeur pour le pâturage : productivité, valeur nutritive, courbe de croissance, charge animale à préconiser. Des recommandations pour l'entretien ou l'amélioration des végétations sont proposées et des hypothèses d'évolution sont formulées. L'établissement de cette classification est une étape déterminante pour la poursuite de travaux de recherches sur la cartographie de ces végétations, par utilisation des images satellitaires dans une optique de gestion des espaces pastoraux, et sur l'évolution des végétations d'altitude.
\end{abstract}

Alpes / pâturage de montagne / typologie de la végétation / composition floristique / valeur pastorale / production végétale / diagnostic

Summary - Agro-ecological diagnosis of the vegetation on dairy cow mountain pastures in the French northern Alps: development and utilization of a simplified typology. Ensuring perennial growth of dairy cow mountain pastures in the French northern Alps is particularly dependent upon good exploitation of mountain resources. A good knowledge of these resources is essential for appropriate management. A typology of vegetation on such pastures has been developed aiming at an agro-ecological diagnosis. It is based upon 220 linear surveys, sampled from 7 summer pastures representative of the area under study. Multivariate analysis of these data, including correspondence analysis followed by hierarchical clustering resulted in the selection of 11 types and 25 subtypes of vegetation. This typology serves various purposes. It is a guide for a simplified identification of pastoral resources which is simple enough to be used by individuals with no specific expertise in flora. The key for identification of vegetation types is based upon a joint assessment of different criteria: morphology (ie plant height), dominant or characteristic species (25 for the 11-type simplified typology), environmental conditions, or grazing and fertilizing practices. This classification can be extended to all mountain pastures in the northern part of the French Alps. It is a tool for pastoral diagnosis, since each vegetation type is defined by its grazing capacity, in terms of productivity, nutritive value, growing rate and stocking rate. Recommendations have been made to maintain or improve native pasture vegetation, and evolutionary hypotheses have been put forward. Establishment of this typology is an essential step for future research on vegetation dynamics analysis and also for vegetation mapping via remote sensing techniques, in view of rangeland managment in mountainous areas. 


\section{INTRODUCTION}

Dans les Alpes du Nord, la majorité des systèmes d'exploitation de montagne est basée sur une production fromagère de qualité, assurée en particulier par la production de lait en alpage. La pérennité de ces alpages à vaches laitières dépend d'une bonne gestion de leurs ressources pastorales, qu'il convient de bien connaître afin de les exploiter correctement. Or, ces alpages présentent une grande diversité de groupements végétaux liée aux variations des conditions de milieu (Legros et al, 1987; Dorioz et Van Oort, 1991), et aux différences de mode d'utilisation (Dubost et Bornard, 1987). Cette diversité des types de végétation rencontrés s'accompagne d'une richesse spécifique élevée : de nombreuses plantes sont présentes en un même lieu, et bon nombre ne se rencontrent qu'en altitude. La reconnaissance de la végétation dans l'optique d'évaluer les ressources d'un alpage représente un exercice délicat qui nécessite des connaissances et des méthodes appropriées.

D'importants travaux synthétiques portent sur la végétation des Alpes du Nord (Ozenda, 1985; Richard et Pautou, 1982); leurs approches biogéographiques et phytosociologiques permettent d'effectuer des zonations à petite échelle, mais les groupements phytosociologiques peuvent recouvrir des réalités agronomiques ou pastorales très différentes. Les approches pastorales nécessitent de définir des unités phytoécologiques élémentaires, les faciès, homogènes au plan de la qualité pastorale. Cela peut être obtenu par l'analyse de la composition floristique quantifiée et impose une échelle de travail comprise entre le 1/5000 et le 1/10 000 (Delpech, 1979; Jouglet et Jacquier, 1976; Dubost et Jouglet, 1983).

Les objectifs qui président à l'élaboration de cette typologie de la végétation appliquée aux alpages des Alpes du Nord sont de 3 ordres. Le but principal est de fournir un guide de reconnaissance de la végétation, assez simple pour être utilisé par des non-spécialistes de la flore tout en étant assez précis pour rendre compte de la qualité pastorale et de la diversité existante; cette simplification conduit à des regroupements de faciès. Le second objectif de cette classification est sa possibilité d'extrapolation au niveau régional. Pour les pâturages d'altitude, certaines typologies ont été élaborées à partir de données recueillies sur des sites localisés, un alpage ou une petite vallée de 100 à 10000 ha (Dorée, 1976; Philippe, 1982; Vertès, 1983;
Costa et al, 1990); d'autres ont été établies à partir de références dispersées au sein d'un massif montagneux de quelques centaines à un millier de $\mathrm{km}^{2}$ (Delpech, 1970; Lacoste et Roux, 1971; Jouglet et Jacquier, 1976; de Montard et Gachon, 1978). À l'exemple des travaux de Dietl et al (1981) qui s'appliquent aux différents massifs alpins de la Suisse, la typologie proposée dans ce document s'adresse aux Alpes externes du Nord, ensemble biogéographique de grande dimension, réunissant plusieurs massifs montagneux (> $4000 \mathrm{~km}^{2}$ ). Afin que l'extrapolation de nos références puisse se réaliser avec une probabilité élevée de réussite au niveau de la région, la classification a été élaborée à partir d'un grand échantillon de stations, dispersées sur plusieurs alpages de la zone d'étude, et plus précisément sur des alpages laitiers, système pastoral traditionnel et dominant de la zone étudiée. Enfin, le dernier objectif de la typologie proposée est qu'elle permette de comprendre le mode de répartition spatiale des types de végétation et de porter un jugement opérationnel sur les ressources pastorales et leurs conditions d'utilisation, afin d'apporter une aide au diagnostic agropastoral de terrain.

\section{MATÉRIEL ET MÉTHODE}

\section{Zone de validité}

La zone d'étude s'étend sur le domaine pastoral des 2 départements de Haute-Savoie et Savoie, région traditionnellement laitière, où l'on trouve aujourd'hui 700 alpages laitiers. On s'est limité à l'ensemble biogéographique des Alpes externes Nord occidentales (Ozenda, 1985), qui constitue la zone de validité de la typologie (fig 1). Cette zone est caractérisée au niveau du climat par :

- des précipitations annuelles importantes : en moyenne $1600 \mathrm{~mm}$ en fond de vallée, sauf en BasseMaurienne et en Basse-Tarentaise (1 $100 \mathrm{~mm})$. Ces précipitations augmentent avec l'altitude; elles dépassent $2800 \mathrm{~mm}$ sur les plus hauts sommets;

- des précipitations estivales élevées (> $400 \mathrm{~mm}$ en fond de vallée); elles assurent en montagne une alimentation hydrique très satisfaisante pour la production de l'herbe;

- un enneigement important.

Dans ce vaste ensemble comprenant des massifs calcaires et cristallins, le domaine pastoral laitier a tendance à se concentrer, aujourd'hui, presque exclusivement sur les milieux sédimentaires carbonatés, aux conditions édaphiques et topographiques plus favorables aux herbages et à l'exploitation pastorale. 


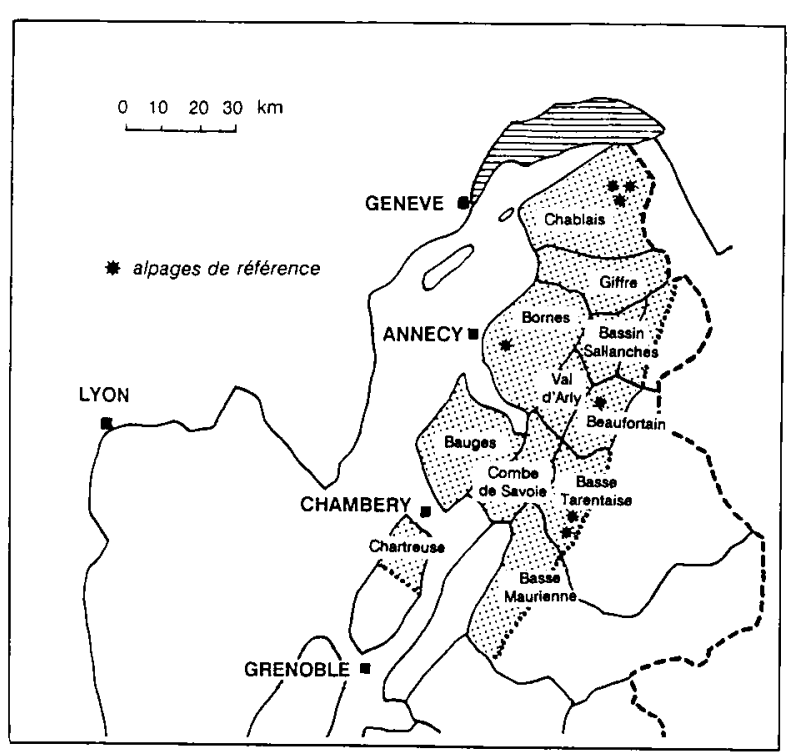

Fig 1. Zone de validité de la typologie.

Dans l'ensemble des Alpes externes du Nord, les alpages laitiers sont situés en majeure partie dans l'étage subalpin et la partie supérieure de l'étage montagnard. Ces alpages résultent de défrichements très anciens. Ils retournent plus ou moins rapidement vers un état forestier après abandon du pâturage. La végétation est abondante, et sa répartition fortement dépendante non seulement des conditions de milieu, mais également des conditions d'exploitation par l'homme. On les trouve également en faible proportion dans l'étage alpin, au-dessus de la limite supérieure des forêts. La végétation est alors composée essentiellement de groupements herbacés, stables, non ou peu dépendants de l'action humaine; leur répartition dépend tout particulièrement des conditions d'enneigement, elles-mêmes fortement liées à la microtopographie et à l'exploitation du versant.

\section{Choix des stations}

Pour l'élaboration de cette typologie, l'échantillon était constitué de 220 stations réparties sur 7 alpages qui ont servi de référence (fig 1). Ces alpages situés dans la région biogéographique précédemment citée ont été retenus pour saisir la diversité régionale, au niveau des conditions de milieu et des pratiques.

L'échantillon est représentatif des grands modes d'exploitation des alpages laitiers : "petite montagne», «moyenne montagne», traditionnellement exploitées de façon intensive, et les «grandes montagnes» extensives; auxquelles s'ajoutent de nouveaux modes, les petites et moyennes montagnes extensives (Dubost et Bornard, 1987).

Les alpages de référence couvrent la diversité des roches mères sédimentaires rencontrées dans les
Alpes externes du Nord : calcaires durs, calcaires marneux, calcschistes et flyschs de diverses sortes qui peuvent engendrer des sols variés acides, calciques, ou plus rarement calcaires (Legros et al, 1987). Ces différences de sols peuvent avoir des conséquences sur la nature de la végétation et sur la dynamique de celle-ci.

\section{Informations utilisées}

\section{La végétation}

\section{Composition floristique}

Les informations sont issues de la méthode des relevés linéaires, qui permet de connaître la composition quantitative d'un peuplement végétal (Daget et Poissonet, 1971). La technique initiale du relevé à été adaptée : la présence des espèces est recensée à la verticale de 50 points de lecture, le long d'une ligne de mesure de $20 \mathrm{~m}$; la longueur de ce segment estompe les effets des microvariations de la station et évite les pertubations de lecture d'un point à l'autre.

Pour l'élaboration de la typologie nous avons utilisé l'information de base contenue dans un relevé de végétation, de la façon suivante :

- les contributions spécifiques des espèces ont constitué les variables actives des analyses multifactorielles qui ont permis la construction des types;

- les groupes d'espèces dominantes, les espèces indicatrices, la liste complète des espèces et l'indice de valeur pastorale ont permis l'interprétation des types.

\section{Production et valeur nutritive}

Au cours des différentes recherches sur les alpages laitiers, des mesures de la production de matière sèche du premier cycle ont été effectuées dans 115 stations différentes, dont la composition floristique est connue.

Ces mesures sont toutes effectuées à la minitondeuse dans 2 types de conditions:

- dans des parcelles $(n=15)$ mises en défens, c'està-dire protégées du pâturage des animaux pendant toute la saison d'estive : les prélèvements à la minitondeuse ( 3 bandes $0,1 \mathrm{~m} \times 10 \mathrm{~m}$, pour saisir l'hétérogénéité interne du faciès) sont effectués régulièrement de 4 à 8 fois par été au cours du premier cycle, en repérant en même temps le stade phénologique du cortège floristique;

- sur d'autres emplacements, qui correspondent à des relevés de végétation $(n=100)$, hors de tout dispositif, la mesure est effectuée une seule fois dans la saison avant le pâturage des animaux ( 3 bandes de $0,1 \mathrm{~m} \times 10 \mathrm{~m}$ ), en repérant aussi les stades phénologiques des espèces dominantes.

Des analyses ont également été effectuées sur une partie des échantillons, afin de mesurer la valeur nutritive de l'herbe offerte (UFL, PDIE, PDIN). La di- 
gestibilité de la matière organique a été estimée par la méthode enzymatique dite de la «cellulase».

\section{Le milieu}

De nombreux travaux (Legros, 1986; Legros et al, 1987; Dorioz et Party, 1987; Dorioz, 1987; Van Oort et Dorioz, 1991) ont mis en évidence les grands mécanismes qui expliquent une large part des variations dans la répartition spatiale des végétations d'altitude. Il s'agit, d'une part, de la tendance générale à la décarbonatation du matériau pédologique avec pour corollaire l'acidification, et d'autre part, de la sensibilité générale à l'engorgement par accumulation d'eau en excès, ces 2 phénomènes étant plus ou moins accentués selon les modalités stationnelles. Nous avons ainsi retenu pour décrire les conditions de milieu, des variables qualitatives facilement disponibles pour la totalité des stations étudiées :

- altitude (mètres) : < 1 500; 1 500-1 700; 1 7001 900; 1 900-2 100; > 2 100;

- exposition : N-NE; E-SE; S-SO; O-NO; indifférenciée;

- lithologie : calcaire dur, autres terrains sédimentaires;

- macromorphologie : haut de versant; mi-versant; bas de versant; éboulis avec amont calcaire; versant soliflué; morraine glaciaire;

- micromorphologie : butte; creux; mosaïque buttecreux; pente régulière;

- régime hydrique : xéricité affirmée; humidité affirmée; moyen.

\section{Les pratiques}

En raison de l'importance économique des produits laitiers et de la main-d'œuvre requise, les alpages laitiers ont traditionnellement été l'objet de soins d'entretien attentifs. La clé de voûte en était le mode de gestion des restitutions animales par la pâture ou par épandage, soit à partir du chalet qui sert aussi d'étable, soit à partir d'un système de traite itinérante, en plein air, assurant l'équilibre des prélèvements et restitutions sur l'ensemble de l'alpage, appelé "pachonnée» (Dubost et Bornard, 1987). II faut mentionner en outre une tâche d'entretien essentielle : l'évacuation des eaux en excès. Ces deux pratiques d'entretien à long terme des milieux sont aujourd'hui largement abandonnées : la végétation n'en conserve pas moins les traces nettes de leur influence.

En conséquence, pour définir les pratiques, les quatre variables suivantes ont été retenues, leur nombre ayant été limité par le fait que l'information devait être exhaustive et fiable pour l'ensemble des stations prises en compte:

- épandage actuel ou récent de fumure organique et pâturage régulier annuel;

- épandage ancien de fumure organique et pâturage régulier annuel;
- sans épandage, mais pâturage régulier annuel;

- sans épandage, lieu peu pâturé, éloigné ou d'accès difficile.

\section{Traitement des données}

De nombreuses applications (Lacoste et Roux, 1971; Jouglet et Jacquier, 1976; De Montard et Gachon, 1978; Plantureux et al, 1987) ont montré la puissance des méthodes d'analyse multivariée pour le dépouillement de vastes ensembles de données floristiques. Ces méthodes permettent, grâce à la représentation graphique :

- de classer, rapidement et objectivement, différents relevés en groupements végétaux;

- de souligner les liens forts existant entre certaines espèces et les groupements végétaux;

- d'appréhender en première analyse l'évolution générale de ces groupements par l'étude des liens possibles entre la proximité de 2 ensembles et la dynamique de ces ensembles.

Ces traitements statistiques ont été mis en œuvre avec le logiciel ADDAD, version micro-ordinateur.

La première phase de traitement a consisté à traiter 220 relevés provenant des 7 alpages de référence, par analyse factorielle des correspondances (AFC).

Les variables principales, ou «actives», sont constituées par les contributions spécifiques des 105 espèces les plus fréquentes dans l'ensemble de ces relevés. Les contributions spécifiques des 200 autres espèces, les variables du milieu et des pratiques, citées précédemment, constituent les variables supplémentaires, ou «inactives»: elles n'entrent pas en compte dans la formation des axes factoriels, mais elles sont projetées dans la représentation graphique des plans factoriels aux fins d'interprétation de la signification des axes.

Les premières analyses factorielles ont permis d'individualiser 5 groupes de relevés représentatifs de types de végétation correspondant à des conditions écologiques bien tranchées ou extrêmes: aulnaies, marécages, landes, pelouses maigres acidophiles et pelouses nivales de l'étage alpin; ces végétations sont en général facilement identifiables sur le terrain ou par tri empirique des données, grâce à des différences évidentes de composition floristique (Dubost et Bornard, 1985). II restait alors à faire des partitions dans le vaste ensemble des formations herbacées les plus intéressantes au point de vue pastoral : ce fut la phase principale des traitements.

Cette phase a porté sur l'ensemble des 7 alpages; elle a été concentrée uniquement sur les formations qualifiées de pelouses grasses, de pelouses moyennes et de pelouses intermédiaires, soit 155 relevés de végétation. Ces relevés ont fait l'objet successivement d'une nouvelle AFC et d'une classification ascendante hiérarchique $(\mathrm{CAH}$, méthode des voisins réductibles). 
Ce dernier enchaînement a permis d'aboutir à la classification de la végétation, présentée en détail dans la partie suivante.

\section{RÉSULTATS}

\section{Interprétation des analyses de données}

Dans la dernière analyse factorielle, les quatre premiers axes ont respectivement un taux d'inertie de $6,7 \%, 5,1 \%, 4,7 \%$ et $4,3 \%$. Seuls les axes 1 et 2 ont été interprétés (fig 2). Sur l'axe 1, les espèces qui ont les plus fortes contributions, à droite, sont les espèces exigeantes en substances nutritives (Landolt, 1977) : Festuca pratensis, Ranunculus aconitifolius, Dactylis glomerata, Geranium sylvaticum, Deschampsia cespitosa, Polygonum bistorta; elles s'opposent, du côté gauche aux espèces oligotrophes : Nardus stricta, Plantago alpina, Polygonum viviparum. Cette opposition se retrouve dans les pratiques pastorales associées aux stations, du côté droit les stations reçoivent ou ont reçu des épandages de fumure organique importants, tandis que du côté gauche les stations ne sont jamais fumées et sont peu pâturées.

On peut considérer que l'axe 1 représente un gradient de richesse du milieu en éléments nutritifs et oppose systématiquement les pelouses grasses d'un côté (coordonnées positives), aux formations des milieux acidifiés de l'autre (pelouses maigres, pelouses nivales).

Sur l'axe 2, les espèces les plus contributives en bas sont Dactylis glomerata, Carlina acaulis, Thymus serpyllum, qui sont des espèces fréquentes sur les sols secs; en haut de l'axe 2, Rumex alpinus, Ranunculus aconitifolius, Deschampsia cespitosa, Phleum alpinum, Ligusticum mutellina, Plantago alpina sont des espèces indicatrices d'humidité ou de fraîcheur

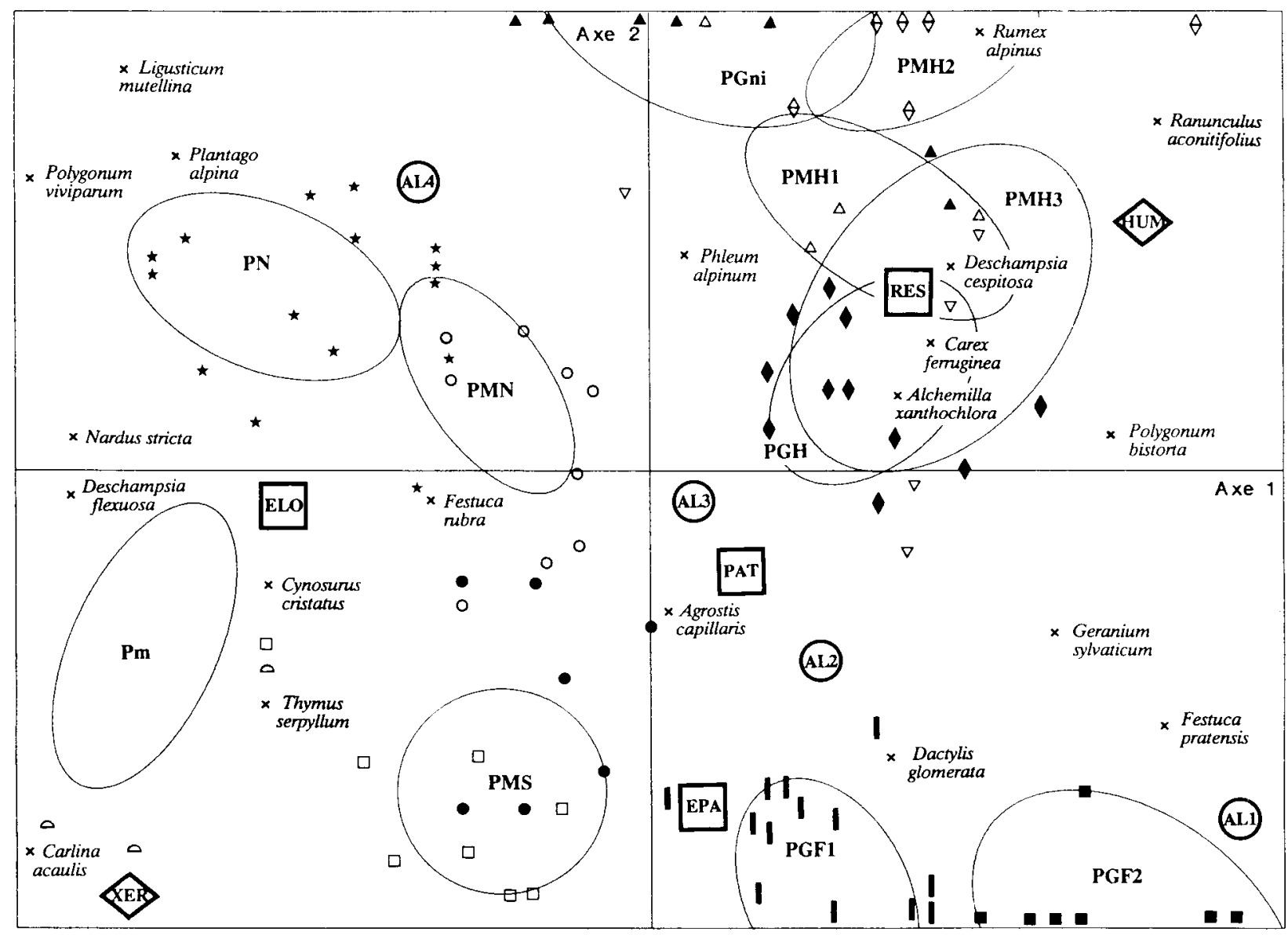

Fig 2. Représentation simplifiée des classes retenues de la partition de la CAH par rapport aux axes 1-2 de l'AFC. Types de pelouses : I Grasses fraîches $\left(P_{G F}\right)$; Grasses fraîches montagnardes $\left(P G F_{2}\right)$; $\bullet$ Grasses neutres (PGN); $\square$ Grasses humides $(\mathrm{PGH}) ; \Delta$ Grasses nitrophiles (PGni); O Moyennes neutres (PMN); Moyennes sèches (PMS); $\Delta$ Moyennes humides $\left(\mathrm{PMH}_{1}\right) ; \theta$ Moyennes humides $\left(\mathrm{PMH}_{2}\right)$; $\nabla$ Moyennes humides $\left(\mathrm{PMH}_{3}\right)$; $\star$ Nivales $(\mathrm{PN})$; ○ Maigres $(\mathrm{Pm})$. Variables supplémentaires : $\square$ Pratique/restitutions animales : EPA : épandage actuel ou récent de fumure organique; RES : épandage ancien de fumure organique; PAT : sans épandage, pâturage régulier; ELO : sans épandage, peu pâturé, éloigné; $O$ Topographie : $A L_{1}:<1500 \mathrm{~m} ; A L_{2}: 1500-$ $1700 \mathrm{~m} ; \mathrm{AL}_{3}: 1700-1900 \mathrm{~m} ; \mathrm{AL}_{4}: 1900-2100 \mathrm{~m} ;$ Régime hydrique : HUM : humidité affirmée; XER : xéricité affirmée. 
très marquée et dont les dernières sont révélatrices d'un enneigement prolongé.

II semble que l'axe 2 soit plus complexe, il représente une combinaison d'un gradient d'humidité et d'un gradient d'altitude, en opposant les milieux humides et haut en altitude (coordonnées positives), aux milieux secs et bas en altitude.

La classification ascendante hiérarchique (CAH) est effectuée à partir des coordonnées factorielles des relevés sur les quatre premiers axes de I'AFC précédente. L'examen de l'histogramme des indices de niveau de hiérarchie a permis de décider du niveau de coupure après une chute significative de celui-ci. Cette coupure a abouti à une partition de l'ensemble des relevés en 15 classes, schématisée sur la figure 3. Une vérification de la composition de ces classes, relevé par relevé, a permis de conclure à la cohérence pastorale de 11 classes parmi les 15 , à partir des critères essentiels suivants : homogénéité des conditions écologiques stationnelles, valeurs pastorales voisines, nombre minimal de relevés.

Ces 11 classes déterminées de façon automatique par la $\mathrm{CAH}$ ont constitué les types définitifs retenus dans la typologie proposée (à l'exception d'une seule, sur laquelle on reviendra); elles sont représentées sur le diagramme de la figure 2, sous forme d'ellipses d'inertie.

Par ailleurs, nous avons été amenés a subdiviser l'une des 11 classes citées précédemment par une interprétation non issue de traitement statistique, afin de distinguer des pelouses moyennes sèches (calcicoles ou acidophiles), d'un groupe qualifié de "gras-neutre», type intermédiaire entre les pelouses grasses et

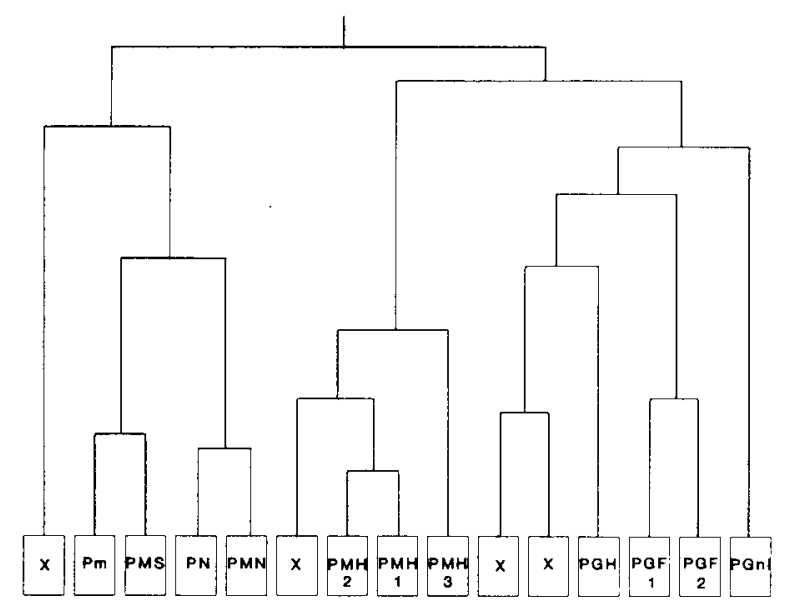

Fig 3. Représentation schématique de l'arbre de la classification ascendante hiérarchique $(\mathrm{CAH})$. moyennes, révélant des arrières-effets d'une fertilisation ancienne abandonnée actuellement.

\section{Organisation d'ensemble de la typologie}

L'ensemble des analyses précédentes a permis de proposer un organigramme synthétique des types de végétation identifiés (fig 4). Dans la construction de ce schéma, on a privilégié les aspects pédagogiques afin d'aboutir à une clé de détermination des types de végétation, quitte à s'éloigner parfois de l'arbre hiérarchique proposé par les méthodes statistiques.

Pour des raisons de reconnaissance pratique par les agents de développement, on a retenu une typologie finale à 2 niveaux :

- celle de niveau 1, ou "simplifiée" est constituée de 11 types issus directement des analyses multivariées (soit les classes finales, soit des agrégations de classes);

- celle de niveau 2, ou "détaillée", est constituée de 25 types; elle a été élaborée soit directement par l'analyse de données, soit par une identification complémentaire issue de l'expérience de terrain afin de proposer quelques soustypes avec une homogénéité phytoécologique plus grande, exigence quelquefois nécessaire dans des applications agronomiques.

On distingue tout d'abord 3 grandes catégories de formations végétales:

- les milieux où s'est développée une végétation ligneuse : ce sont essentiellement les landes ou les brousses d'Alnus viridis;

- les milieux affectés par un excès d'eau temporaire ou permanent, aisément identifiables : ils hébergent des peuplements herbacés très particuliers : ce sont les zones humides;

- des peuplements dominés par la strate herbacée, sans excès d'eau, que nous qualifierons de pelouses : contrairement aux précédents dont l'intérêt pour le pâturage est souvent marginal, ils représentent l'essentiel des ressources pastorales d'un alpage, aussi la typologie proposée met particulièrement en évidence leurs différences de qualité pastorale. Pour faciliter la différenciation des types de pelouses, des critères simples de description physionomique (hauteur de la strate, densité du couvert) ont été rajoutés.

Au sein de la catégorie pelouses on discrimine deux grands ensembles :

- l'ensemble des pelouses ayant parmi leurs espèces dominantes 2 graminées, de qualité 


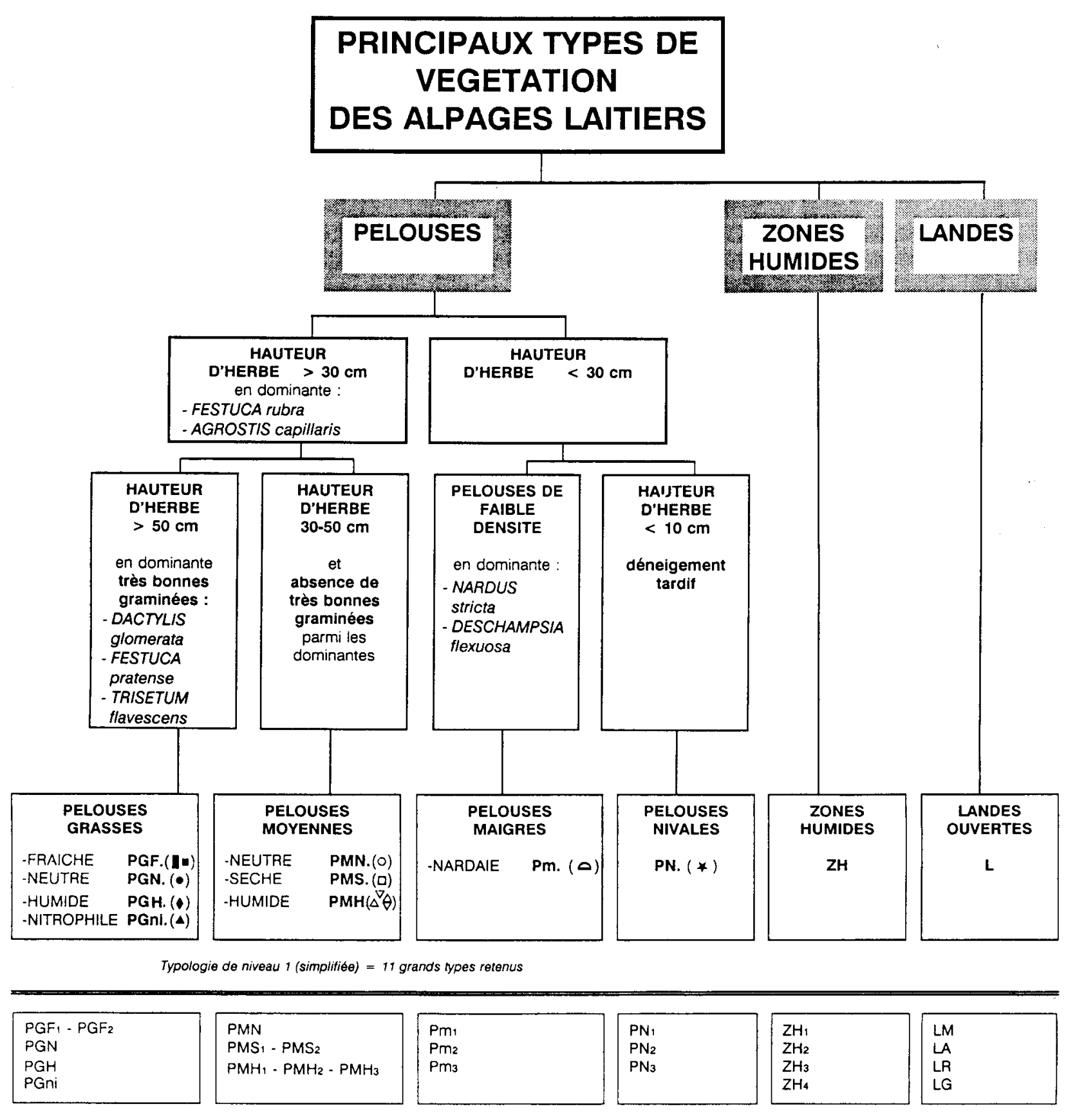

Typologie de niveau 2 (détaillée) $=25$ types identifiés (ou sous-types des grands types précédents)

Fig 4. Schéma de l'organisation d'ensemble de la typologie.

moyenne pour le pâturage, mais qui jouent un rôle très important dans la caractérisation des herbages d'altitude : il s'agit de Festuca rubra et d'Agrostis capillaris; ces pelouses se présentent comme les plus hautes $(>30 \mathrm{~cm}$, en pleine végétation), plus ou moins denses : elles sont représentatives de milieux régulièrement parcourus et entretenus par les animaux, ce sont les catégories grasses et moyennes;

- l'ensemble des autres pelouses, dont la hauteur d'herbe est inférieure à $30 \mathrm{~cm}$ en pleine vé- gétation, dominées par d'autres espèces de graminées : l'ensemble est assez hétérogène et les contraintes de milieu, très marquées, jouent tantôt sur la qualité tantôt sur la quantité de la matière sèche; ce sont les catégories nivales et maigres.

\section{Pelouses grasses}

Ce sont des pelouses à phytomasse herbacée très abondante et qui comportent des graminées 
de bonne qualité fourragère parmi les espèces dominantes, en plus de Festuca rubra et d'Agrostis capillaris : Dactylis glomerata, Festuca pratensis, Poa pratensis, ou Cynosurus cristatus. En référence à l'abondance et à la qualité de la biomasse pour le pâturage, nous qualifierons ce sous-ensemble de pelouses grasses: elles sont représentatives de milieux riches en éléments nutritifs. Cette richesse provient de conditions naturelles soit très favorables, soit favorables (sans excès d'humidité ni de sécheresse, sols non fortement acidifiés) auxquelles sont venus s'ajouter des soins particuliers dans l'exploitation du pâturage : ces pelouses ont en effet bénéficié d'une concentration des fertilisants organiques, le plus souvent par épandage, ou lors du pâturage régulier. Les soins attentifs réservés aux alpages laitiers mais aussi l'abondance des précipitations et des matériaux carbonatés expliquent le développement tout particulier de ce type de pelouses dans cette région.

Ces pelouses grasses peuvent être décomposées, suivant leur régime hydrique en 3 types: humide, frais, et "neutre» (sécheresse modérée), auxquels s'ajoutent un quatrième type : nitrophile, correspondant à des milieux avec excès de restitutions (tableau I).

\section{Pelouses moyennes}

Ces pelouses à phytomasse herbacée moins abondante que les précédentes ne comportent pas de très bonnes graminées fourragères parmi les dominantes. II s'agit de milieux régulièrement parcourus, mais où les conditions naturelles ou d'exploitation introduisent des facteurs limitants, avec une grande variété de nuances et combinaisons possibles : par exemple, restitution satisfaisante des éléments prélevés au pâturage mais sensibilité à la sécheresse, ou, à l'inverse, conditions édaphiques satisfaisantes, mais niveau de restitution insuffisant, etc... II est cependant frappant de constater que, malgré toutes les nuances de conditions stationnelles (milieu et pratiques), ces pelouses sont la plupart du temps nettement dominées par Festuca rubra et Agrostis capillaris. Sur les alpages laitiers de cette région elles représentent le sous-ensemble des pelouses moyennes, en qualité et quantité; ailleurs, soit en raison d'une prédominance des matériaux cristallins, soit en l'absence de traditions comparables d'entretien des herbages, soit en raison d'un climat plus rude (massifs moins arrosés, ou alpages situés plus hauts, dans l'étage alpin), de telles végétations représenteraient les meilleurs pelouses.

Ce deuxième sous-ensemble est subdivisé en 3 grands types, suivant le régime hydrique : humide, «neutre" (sécheresse modérée), et sec. Les types sec et humide comportent respectivement 2 et 3 sous-types suivant la nature du sol ou le degré d'humidité (tableau I).

\section{Pelouses nivales}

Ce sont des pelouses rases à développement tardif situées dans des dépressions et sur des replats à longue période d'enneigement. Par extension de la terminologie utilisée pour désigner les modes de l'étage alpin, nous les qualifierons de «nivales».

La phytomasse aérienne mesurée de ces pelouses est peu importante en quantité, mais elle est très appétente compte tenu des espèces végétales en présence et du développement tardif de la végétation. Celle-ci est dominée par des plantes à rosette (Leontodon sp, Plantago alpina, Trifolium alpinum).

La longueur de la période d'enneigement différencie 3 types, où les contraintes naturelles sont de moins en moins fortes : les pelouses nivales alpines ( $>2000 \mathrm{~m}$ d'altitude), les combes subalpines (1 600-2 $000 \mathrm{~m}$ ), et les pelouses prénivales (secteur déneigé tardivement, 1 800-2 000 m) (tableau II).

\section{Pelouses maigres acidophiles}

Elles sont rassemblées dans une seule catégorie, décomposable en 3 types. Leur caractéristique commune est de posséder Nardus stricta dans la liste des espèces dominantes, à côté d'espèces franchement caractéristiques de milieux pauvres et acides. Le terme général de pelouses maigres ne se limite pas à la Nardaie pure et comporte de nombreuses variantes. Un premier type est dominé par Deschampsia flexuosa, bien représentative de ces milieux acidifiés; les deux autres au contraire par des espèces de qualité pastorale bonne ou moyenne (Plantago alpina, ou Festuca rubra ou Phleum alpinum). En outre, figurent également en dominantes d'autres acidophiles notoires, telles que Potentilla Tormentilla, Gentiana purpurea, Luzula sylvatica, Geum montanum (tableau II). 
Tableau I. Composition florisitique des types de végétation des alpages laitiers des Alpes du Nord : contributions spécifiques moyennes (\%) des espèces dominantes et de quelques espèces indicatrices.

\begin{tabular}{|c|c|c|c|c|c|c|c|c|c|c|c|}
\hline \multirow{3}{*}{$\begin{array}{l}\text { Grandes catégories } \\
\text { Typologie simplifiée } \\
\text { Typologie détaillée * }\end{array}$} & \multicolumn{5}{|c|}{ Grasse } & \multicolumn{6}{|c|}{ Moyenne } \\
\hline & \multicolumn{2}{|c|}{ Fraîche } & \multirow{2}{*}{$\begin{array}{l}\text { Neutre } \\
P G N\end{array}$} & \multirow{2}{*}{$\begin{array}{l}\text { Humide } \\
P G H\end{array}$} & \multirow{2}{*}{$\begin{array}{l}\text { Nitro } \\
\text { PGni }\end{array}$} & \multirow{2}{*}{$\begin{array}{l}\text { Neutre } \\
P M N\end{array}$} & \multicolumn{2}{|c|}{ Sèche } & \multicolumn{3}{|c|}{ Humide } \\
\hline & $P G F_{1}$ & $P G F_{2}$ & & & & & $P M S_{1}$ & $\mathrm{PMS}_{2}$ & $\mathrm{PMH}_{1}$ & $\mathrm{PMH}_{2}$ & $\mathrm{PMH}_{3}$ \\
\hline Festuca rubra & 16,7 & 12,1 & 19,8 & 19,3 & 15,1 & 20,0 & 19,8 & 11,0 & 12,9 & 9,9 & 9,5 \\
\hline Agrostis capillaris & 15,5 & 10,6 & 16,3 & 9,2 & 4,3 & 11,8 & 8,6 & 5,5 & 6,9 & 5,4 & 6,3 \\
\hline Dactylis glomerata & 13,1 & 13,6 & 3,5 & 2,8 & - & 1,2 & 2,5 & - & 1,0 & 1,8 & 4,0 \\
\hline Alchemilla xanthochlora & 7,1 & 7,6 & 3,5 & 14,7 & 9,7 & 8,2 & 3,7 & 3,3 & 7,9 & 11,7 & 5,6 \\
\hline Geranium sylvaticum & 4,8 & 3,0 & 1,2 & 1,8 & - & 1,2 & - & - & 1,0 & 2,7 & 4,8 \\
\hline Festuca pratensis & 1,2 & 12,1 & - & 0,9 & - & - & - & - & - & - & - \\
\hline Phleum alpinum & - & - & 4,7 & 9,2 & 14,0 & 4,7 & - & - & 13,9 & 8,1 & - \\
\hline Thymus serpyllum & - & - & 3,5 & - & - & 1,2 & 3,7 & 5,5 & - & - & - \\
\hline Cynosurus cristatus & 1,2 & - & 2,3 & 0,9 & - & 1,2 & 1,2 & - & - & - & 0,8 \\
\hline Deschampsia cespitosa & - & - & - & 10,1 & 1,1 & 3,5 & - & - & 13,9 & 9,9 & 4,0 \\
\hline Rumex alpinus & 1,2 & - & - & 0,9 & 19,4 & - & - & - & - & 0,9 & - \\
\hline Ranunculus acris & - & 1,5 & - & 2,8 & 9,7 & 3,5 & - & 2,2 & - & 1,8 & 0,8 \\
\hline Plantago atrata & - & - & 1,2 & 0,9 & - & 1,2 & 2,5 & 2,2 & 1,0 & - & - \\
\hline Phleum pratense & 1,2 & 3,0 & - & 0,9 & - & - & - & - & - & 0,9 & - \\
\hline Leucanthemum vulgare & - & - & - & - & - & 1,2 & 2,5 & 1,1 & - & - & 0,8 \\
\hline Nardus stricta & 1,2 & - & 3,5 & 0,9 & 2,2 & 2,4 & - & 3,3 & - & 0,9 & - \\
\hline Chamaespartium sagittale & - & - & 1,2 & - & - & - & 2,5 & - & - & - & - \\
\hline Ranunculus aconitifolius & - & - & - & - & - & - & - & - & 2,0 & 13,5 & 2,4 \\
\hline Polygonum Bistorta & - & - & - & 0,9 & - & - & - & - & - & 5,4 & - \\
\hline Ligusticum mutellina & - & - & - & - & 1,1 & - & - & - & 5,0 & 1,8 & 1,6 \\
\hline Carex ferruginea & - & - & 1,2 & - & - & - & - & - & 2,0 & - & 11,9 \\
\hline
\end{tabular}

Chiffres en gras : contributions spécifiques $(\%)$ des 5 espèces dominantes de chaque type. * Pelouses grasses : PGF $F_{1}:$ fraîches, $\mathrm{PGF}_{2}$ : fraîches montagnardes, $\mathrm{PGN}$ : neutres, $\mathrm{PGH}$ : humides, $\mathrm{PGni}:$ nitrophiles. Pelouses moyennes : PMN : neutres, $\mathrm{PMS} \mathrm{S}_{1}$ : sèches calcicoles, $\mathrm{PMS}_{2}$ : sèches acidophiles, $\mathrm{PMH}_{1}, \mathrm{PMH}_{2}, \mathrm{PMH}_{3}$ : humides.

Ces pelouses peuvent représenter des quantités de matière sèche moyennes, mais sont toujours de qualité fourragère médiocre.

\section{Les zones humides}

Elles désignent un grand type assez hétérogène au sein duquel il faut distinguer 4 sous-types, selon que l'excès d'eau est temporaire ou permanent (tableau II) :

- les pelouses à Molinia caerulea;

- les pelouses à Ranunculus aconitifolius;

- les pelouses à Caltha palustris et Ranunculus aconitifolius;

- les marécages à divers Carex.

Toutes ces végétations sont pauvres en espèces fourragères.

\section{Les landes}

Ces végétations représentent une dégradation de la situation des pelouses maigres, en raison d'une pression pastorale très faible, voire nulle depuis plusieurs décennies ou à cause de conditions stationnelles très défavorables (sols acides ou très acides). Il est possible de distinguer quatre sous-types en fonction de l'espèce ligneuse dominante : Vaccinium Myrtillus, Vaccinium uliginosum, Rhododendron ferrugineum ou Juniperus nana. Sous la strate ligneuse, la pelouse est du type pelouse maigre acidophile.

\section{Les ressources pastorales}

Après avoir identifié chacun des types, l'un des objectifs de cette typologie est de caractériser les ressources disponibles afin de déboucher sur des recommandations en matière de chargement et de plan de pâturage à court terme; les différents indicateurs de production enregistrés permettent de prévoir les ressources pastorales de chacun des types de végétation. 
Tableau II. Composition floristique des types de végétation des alpages laitiers des Alpes. du Nord : contributions spécifiques moyennes (\%) des espèces dominantes et de quelques espèces indicatrices (suite).

\begin{tabular}{|c|c|c|c|c|c|c|c|c|c|c|c|c|c|c|}
\hline \multirow{2}{*}{$\begin{array}{l}\text { Grandes catégories } \\
\text { Typologie simplifiée } \\
\text { Typologie détaillée * }\end{array}$} & \multicolumn{3}{|c|}{$\begin{array}{l}\text { Nivale } \\
\text { Nivale }\end{array}$} & \multicolumn{3}{|c|}{$\begin{array}{l}\text { Maigre } \\
\text { Maigre }\end{array}$} & \multicolumn{4}{|c|}{$\begin{array}{l}\text { Zone humide } \\
\text { Zone humide }\end{array}$} & \multicolumn{4}{|c|}{$\begin{array}{l}\text { Lande } \\
\text { Lande }\end{array}$} \\
\hline & $P N_{1}$ & $P N_{2}$ & $P N_{2}$ & $P m_{1}$ & $\mathrm{Pm}_{2}$ & $P m_{3}$ & $Z H_{1}$ & $\mathrm{ZH}_{2}$ & $\mathrm{ZH}_{3}$ & $\mathrm{ZH}_{4}$ & $L m$ & $L A$ & $L R$ & $L G$ \\
\hline Plantago alpina & 11,6 & 12,2 & 4,6 & 1,1 & 6,8 & 2,0 & - & - & - & - & 2,4 & 1,1 & 0,7 & - \\
\hline Festuca rubra & 9,5 & 16,3 & 14,9 & 8,6 & 9,6 & 14,9 & 3,8 & 8,7 & 3,1 & 3,1 & 8,5 & 9,7 & 2,3 & 4,0 \\
\hline Leontodon pyrenaicus & 9,5 & 2,0 & 4,6 & 6,5 & 3,4 & 2,0 & - & - & 2,0 & - & - & 4,3 & 2,2 & 1,0 \\
\hline Nardus stricta & 6,3 & 3,1 & 3,4 & 10,8 & 13,7 & 3,0 & - & 0,8 & 2,0 & - & 12,2 & 8,6 & 8,3 & 6,0 \\
\hline Trifolium alpinum & 5,3 & - & - & 1,1 & 1,4 & - & - & - & - & - & 2,4 & - & - & - \\
\hline Agrostis capillaris & 2,1 & 9,2 & 2,3 & 5,4 & 4,8 & 4,0 & 5,1 & 10,3 & 4,1 & 3,1 & 2,4 & 1,1 & 0,7 & - \\
\hline Crepis aurea & 2,1 & 7,1 & 1,1 & - & 4,1 & 4,0 & - & - & 1,0 & 0,8 & - & - & - & - \\
\hline Phleum alpinum & 5,3 & 5,1 & 8,0 & 3,2 & 2,1 & 11,9 & - & 0,8 & 1,0 & - & 3,7 & 1,1 & 0,6 & - \\
\hline Leontodon hispidus & 2,1 & 2,0 & 6,9 & - & 1,4 & 1,0 & - & - & 1,0 & - & 1,2 & 1,1 & - & - \\
\hline Potentilla aurea & 3,2 & - & 1,1 & 1,1 & 2,1 & 3,0 & - & - & - & - & 4,9 & 3,2 & 0,5 & 4,0 \\
\hline Ranunculus acris & 4,2 & 1,0 & 5,7 & - & 2,1 & - & - & 1,6 & 3,0 & 1,5 & 3,7 & 1,1 & - & 1,0 \\
\hline Alchimilla xanthochlora & - & 4,1 & 5,7 & - & 5,5 & - & - & 9,5 & 3,1 & 7,7 & - & 1,1 & - & 1,0 \\
\hline Deschampsia flexuosa & 2,1 & - & 1,1 & 28,0 & 0,7 & 3,0 & - & - & - & 3,8 & 13,4 & 14,0 & 10,6 & 10,0 \\
\hline Potentilla erecta & 1,1 & - & - & 8,6 & 0,7 & 2,0 & 6,3 & 1,6 & 1,0 & 3,1 & 3,7 & 1,1 & - & 1,0 \\
\hline Ligusticum mutellina & 3,2 & 1,0 & 1,1 & 5,4 & 5,5 & - & - & - & 1,0 & - & - & - & - & - \\
\hline Geum montanum & 2,1 & 1,0 & - & 1,1 & 4,1 & 3,0 & - & - & - & - & 2,4 & 1,0 & - & 2,0 \\
\hline Gentiana purpurea & - & - & - & - & - & 8,9 & - & - & - & - & - & 2,2 & - & - \\
\hline Luzula sylvatica & - & - & - & - & - & 6,9 & - & 0,8 & - & - & - & - & - & - \\
\hline Arnica montana & - & - & - & 1,1 & 0,7 & 5,0 & - & - & - & - & - & 3,2 & 0,6 & 4,0 \\
\hline Carex sp & - & 1,0 & - & - & 0,7 & - & 36,7 & 15,1 & 14,2 & 13,1 & - & - & - & - \\
\hline Molinia caerulea & - & - & - & - & - & - & 15,2 & 1,6 & - & 2,3 & - & - & - & - \\
\hline Geranium sylvaticum & - & - & 1,1 & - & - & 1,0 & 5,1 & 1,6 & - & 2,3 & - & - & - & - \\
\hline Ranunculus aconitifolius & - & - & - & - & - & - & - & 7,1 & 11,2 & 1,5 & - & - & - & - \\
\hline Mousse sp & 5,3 & 5,1 & 2,3 & 1,1 & 2,7 & 1,0 & 1,3 & 3,2 & 8,2 & 18,5 & - & 5,4 & 26,6 & 3,0 \\
\hline Caltha palustris & - & - & - & - & - & - & - & 4,0 & 6,1 & 4,6 & - & - & - & - \\
\hline Deschampsia cespitosa & - & 2,0 & 2,3 & - & - & - & - & 4,8 & 6,1 & 1,5 & - & - & - & - \\
\hline Carex ferruginea & - & 1,0 & - & - & - & 2,0 & - & 0,8 & - & 9,2 & - & - & - & - \\
\hline Vaccinium Myrtillus & 1,1 & - & - & - & 0,7 & 3,0 & - & - & - & - & 9,8 & 8,6 & 13,6 & 9,0 \\
\hline Vaccinium uliginosum & - & - & - & - & - & 1,0 & - & - & - & - & 2,4 & 12,9 & 5,9 & 4,0 \\
\hline Rhododendron ferrugineum & $7-$ & - & - & - & - & - & - & - & - & - & - & 1,1 & 14,0 & - \\
\hline Anthoxanthum odoratum & - & 1,0 & - & 2,2 & 0,7 & 2,0 & - & 0,8 & - & - & 1,2 & 1,1 & 2,0 & 1,0 \\
\hline Juniperus nana & - & - & - & - & - & - & - & - & - & - & - & - & - & 11,0 \\
\hline
\end{tabular}

Chiffres en gras : contributions spécifiques (\%) des 5 espèces dominantes de chaque type. * Pelouses nivales : $P N_{1}$ : alpines, PN ${ }_{2}$ : combes subalpines, $P N_{3}$ : prénivales. Landes : $L M$ : à myrtille, $L A$ : à airelle, $L R$ : à rhododendron, $L G$ : à genévrier.

\section{La production de matière sèche}

Les niveaux maxima atteints au cours du premier cycle révèlent une bonne hiérarchie entre les grandes catégories de végétation (fig $5 \mathrm{a}$ ) :

- pelouses grasses : 2,5 à 3,5 t/ha;

- pelouses moyennes : 1,5 à 2,5 t/ha;

- pelouses nivales : 0,5 à 1,5 t/ha;

- pelouses maigres : 0,7 à 2 t/ha.

Cependant, en matière pastorale, cet indicateur de productivité est insuffisant, car au pâturage l'animal exerce un certain choix, une partie de la phytomasse produite étant plus ou moins appréciée, l'autre refusée. C'est pour cette raison que diverses méthodes d'estimation de la qualité pastorale d'une végétation ont été proposées.

\section{La valeur pastorale}

La méthode des relevés linéaires permet de quantifier la participation de chaque espèce au peuplement végétal. Moyennant l'attribution d'indices de qualité (indices spécifiques) à chacune de ces espèces, une note de qualité du peuplement végétal appelée «valeur pastorale» peut 
être calculée (Poissonet P, Poissonet J, 1969). II s'agit d'une grandeur sans dimension, pouvant varier en théorie entre 0 et 100 . Cependant, les meilleures valeurs pastorales rencontrées jusqu'alors dans les pâturages d'altitude des massifs français sont de 50-55.

Le classement des types de végétation selon l'indice de valeur pastorale (fig 5b) accentue la hiérarchie établie précédemment par le seul critère de production de matière sèche. La supériorité relative des pelouses grasses par rapport aux pelouses moyennes passe de $+50 \%$ en terme de matière sèche produite à l'hectare, à $+75 \%$ en terme de valeur pastorale. Certaines pelouses dont la production de biomasse aérienne mesurée est relativement importante mais qui contiennent une proportion élevée d'espèces non fourragères $(>60 \%)$ comme les pelouses moyennes humides ou la variante des pelouses maigres acidophiles à Deschampsia flexuosa, sont reclassées à un niveau reflétant mieux leur faible intérêt pastoral, du fait de la non-appétence d'une grande partie de la phytomasse aérienne produite. Les zones humides et les landes ouvertes ont un très faible intérêt pastoral, à cause de leur pauvreté en espèces fourragères; d'ailleurs, en général, les landes sont réservées aux animaux non laitiers.

\section{Charge animale à préconiser}

À partir de l'indice de valeur pastorale, il est possible de calculer, par utilisation de coefficients de conversion, un potentiel théorique fourrager (PTF), puis de déterminer une charge animale à préconiser (CAP) (Cozic; 1987).

Les coefficients de conversion $(K)$ utilisés pour le calcul $P T F(U F L /$ ha $)=V P \times K$ sont issus de la mise en parallèle, sur des sites de référence, de la qualité pastorale de la végétation $(V P)$ et des charges animales (UFL /ha) réellement observées sur ces milieux (Daget et Poissonnet, 1971; Dubost et Jouglet, 1983; Cozic, 1987). Pour les milieux concernés les coefficients sont les suivants:

$-K=55 U F L /$ ha pour tous les types de pelouses grasses et moyennes à l'étage subalpin;

$-K=44 U F L$ ha pour les pelouses maigres, zones humides, landes, et pelouses nivales (sauf variante alpine du sous type $\mathrm{PN}_{1}$ );

$-K=33 U F L$ ha pour les pelouses nivales de l'étage alpin.

La détermination de la charge animale à préconiser prend en compte à la fois les objectifs de production, le niveau de prise de risque des éleveurs face à la variabilité interannuelle et le mode d'exploitation de l'alpage. En fonction de ces caractéristiques, un coefficient réducteur plus ou moins sévère est appliqué au potentiel théorique fourrager. Le «système vaches laitières» exigeant chaque jour de bonnes ou très bonnes performances, réparties régulièrement sur l'été, quelle que soit l'année, il a été admis de fixer la charge à préconiser (CAP) :

- à $60 \%$ du PTF, dans le cas des alpages où il y a au plus deux passages des animaux, et un chargement faible, le système entraînant un gaspillage de l'herbe au début de l'estive;

- à $90 \%$ du PTF, dans le cas d'alpages exploités plus intensivement, où l'on observe plus de deux rotations des vaches laitières dans l'été.

La charge est traduite en nombre de journées de pâturage d'une vache standard par hectare. Les caractéristiques retenues pour une vache standard d'alpage sont les suivantes : poids vif $550 \mathrm{~kg}$, besoins supplémentaires dus aux déplacements $+20 \%$, production laitière $13 \mathrm{~kg} / \mathrm{jour}$.

La classification des types de végétation selon les charges à préconiser, calculées ainsi, est schématisée sur la figure $5 \mathrm{c}$. C'est ce dernier classement, qui montre le plus grand échelonnement entre les catégories de végétation.

\section{Dynamique de croissance}

On a procédé à un ajustement de la productivité en fonction des sommes de températures calculées depuis le déneigement, comme réalisé dans des travaux précédents (Bernard-Brunet et Cozic, 1986), suivant l'altitude des stations, qui s'étale entre 1500 et $2200 \mathrm{~m}$, le déneigement a lieu généralement entre la fin mai et la fin juin, mais on observe de forts écarts entre les années, qui peuvent atteindre 1 mois pour les extrêmes. Plusieurs travaux antérieurs ont montré une liaison entre les stades des espèces et la somme de degrés-jours qui a permis d'établir une liaison statistique pour une cinquantaine d'espèces d'altitude (Jouglet et al, 1982; Lambertin, 1987).

Pour 6 types de végétation $\left(\mathrm{PGF}_{1}, \mathrm{PGN}\right.$, $\mathrm{PGH}, \mathrm{PMN}, \mathrm{PMH}_{3}, \mathrm{PN}_{3}$; voir figure 5 pour la signification des sigles), il a été ainsi possible de décrire la dynamique de croissance en fonction des sommes de degrés-jours ou en fonction des stades de développement des espèces dominantes, critère plus opérationnel. 


\section{a-Production de matière sèche selon les types de végétation}

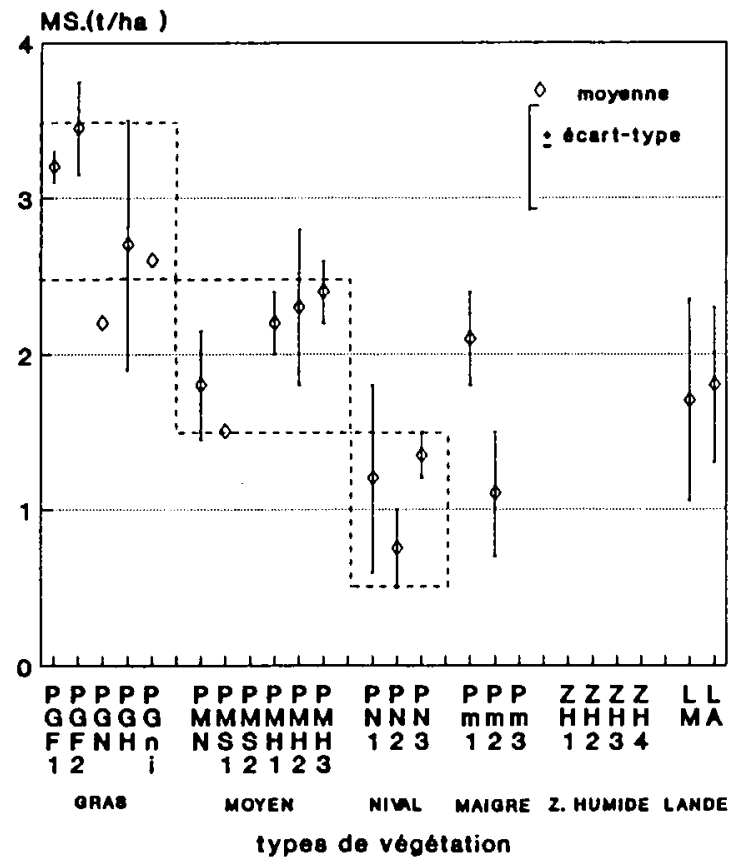

\section{c_Charge à préconiser (jours de påture/ha) selon les types de végétation}

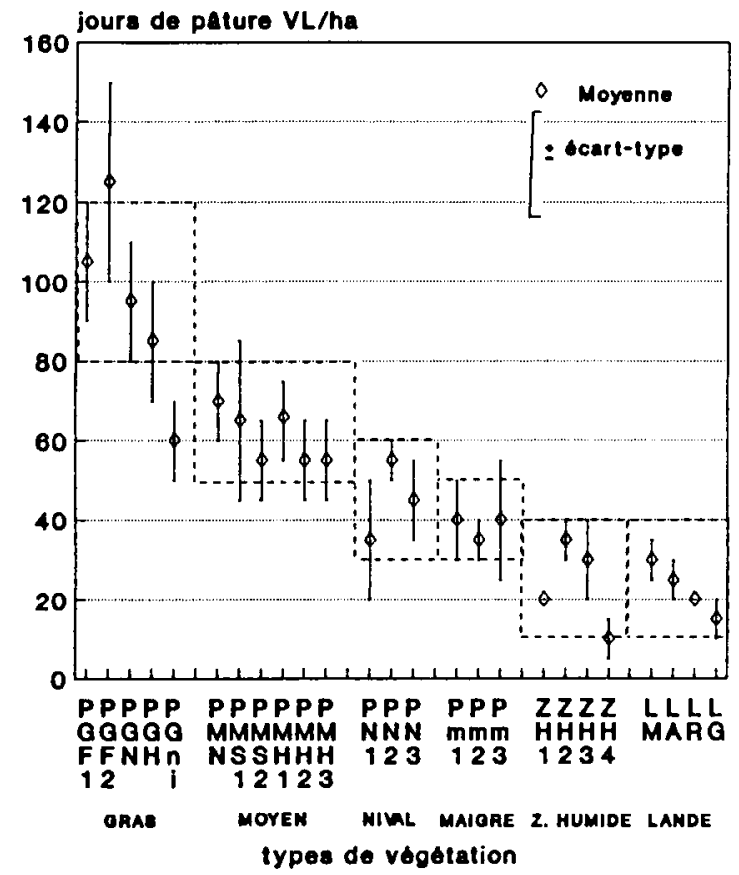

Nous ne donnerons ici que l'exemple de la courbe de croissance des pelouses grasses fraîches (fig 6); les équations d'ajustement pour les autres types de pelouses sont reportées en annexe 1.

\section{b-Indice de Valeur Pastorale selon les types de végétation}

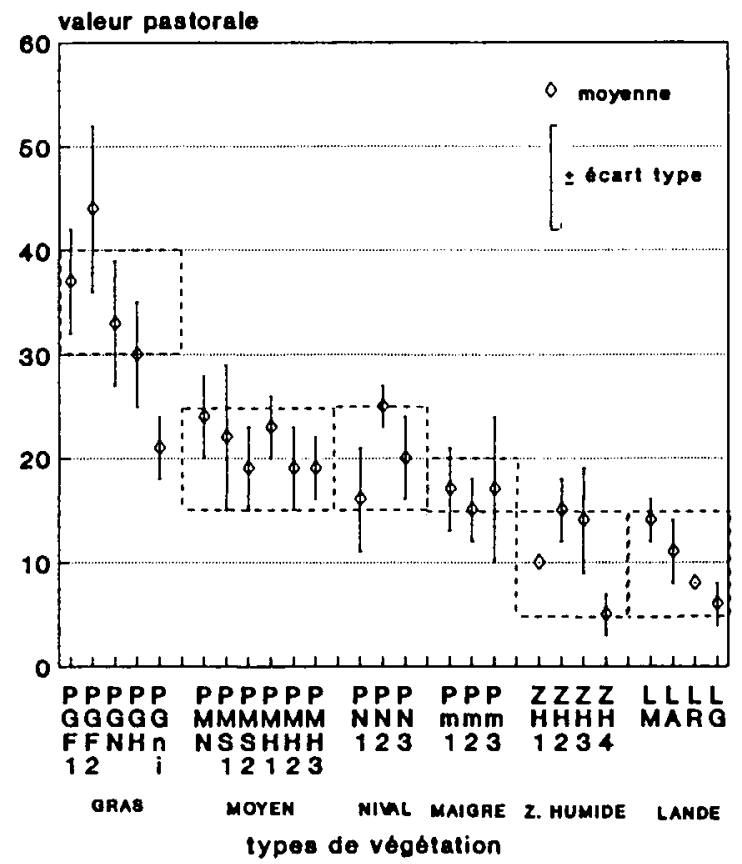

Types de végétation

\begin{tabular}{|c|c|}
\hline PELOUSES GRASSES & PELOUSES MOYENNES \\
\hline \multirow{2}{*}{$\begin{array}{l}\text { PGF1 - Frafches } \\
\text { PGF2 - Fralches monteonardes } \\
\text { PGN - Neutres } \\
\text { PGH - Humides } \\
\text { PGni - Nitrophiles }\end{array}$} & $\begin{array}{l}\text { PMN - Neutroe } \\
\text { PMS1 - Stches calcicolea } \\
\text { PMS2 - Stches acidophiles } \\
\text { PMH1, PMH2, PMH3 - Humldes }\end{array}$ \\
\hline & PELOUSES MAIGRES \\
\hline PELOUSES NIMLES & PM1, PM2, PM3 \\
\hline $\begin{array}{l}\text { PN1 - Alpines } \\
\text { PN2 - Combes subalpines } \\
\text { PN3 - Prd-nivales }\end{array}$ & LANDES \\
\hline ZONES HUMIDES & \multirow{2}{*}{$\begin{array}{l}\text { LM - Myrtille } \\
\text { LA - Airelle } \\
\text { LR : Ahododendron } \\
\text { LQ - Gendvrier }\end{array}$} \\
\hline $\mathrm{ZH1}, \mathrm{ZH2}, \mathrm{ZH3}, \mathrm{ZH4}$ & \\
\hline
\end{tabular}

Fig 5. Valeur pour le pâturage des principaux types de végétation.

D'une manière générale, pour tous les types de végétation, on peut escompter atteindre 40 à $50 \%$ du maximum de production de matière sèche dès le stade mi-montaison des graminées tardives comme Dactylis glomerata ou Festuca 

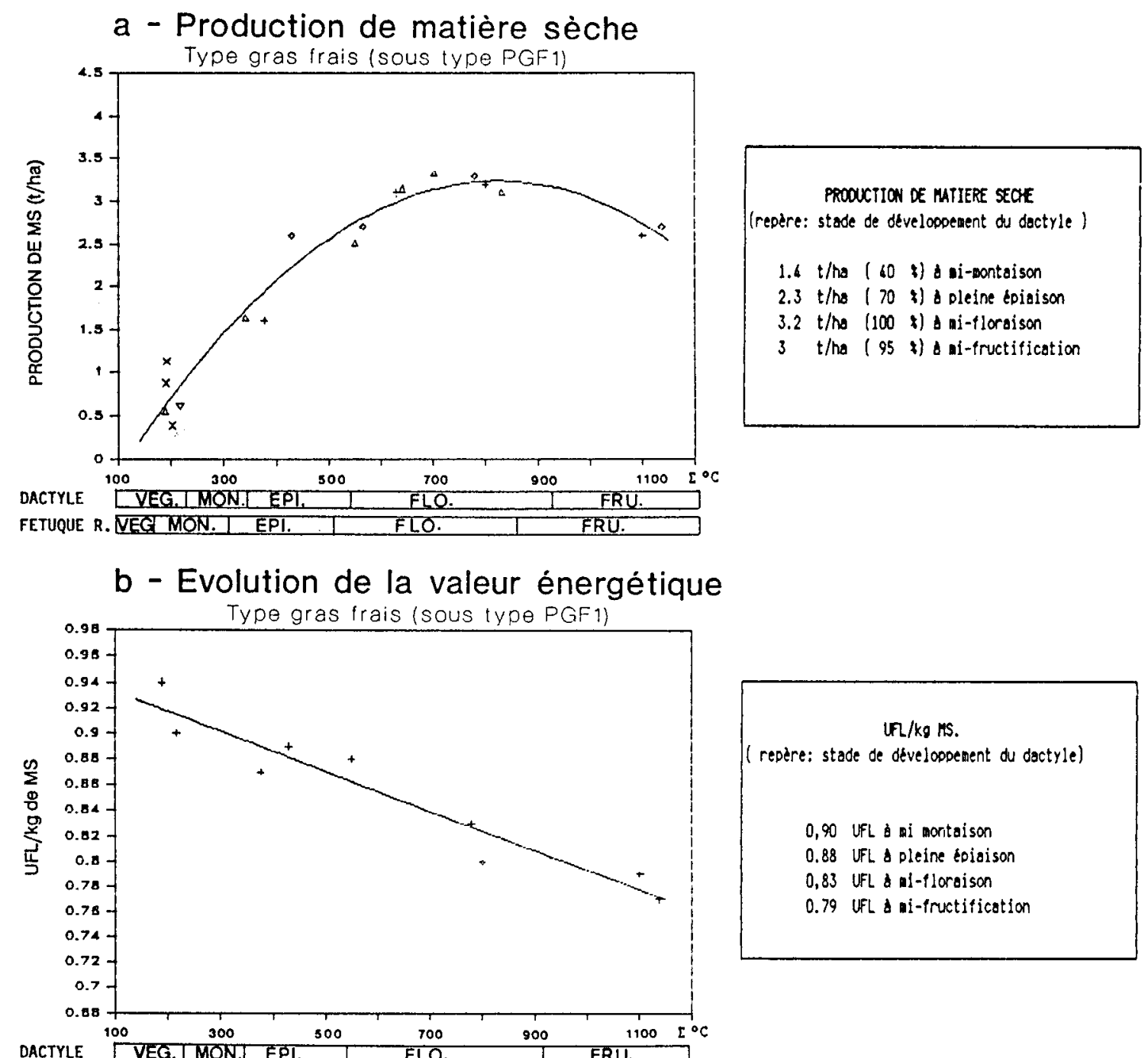

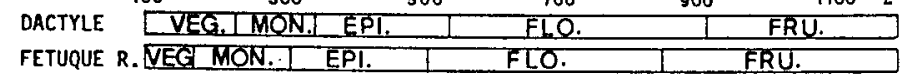
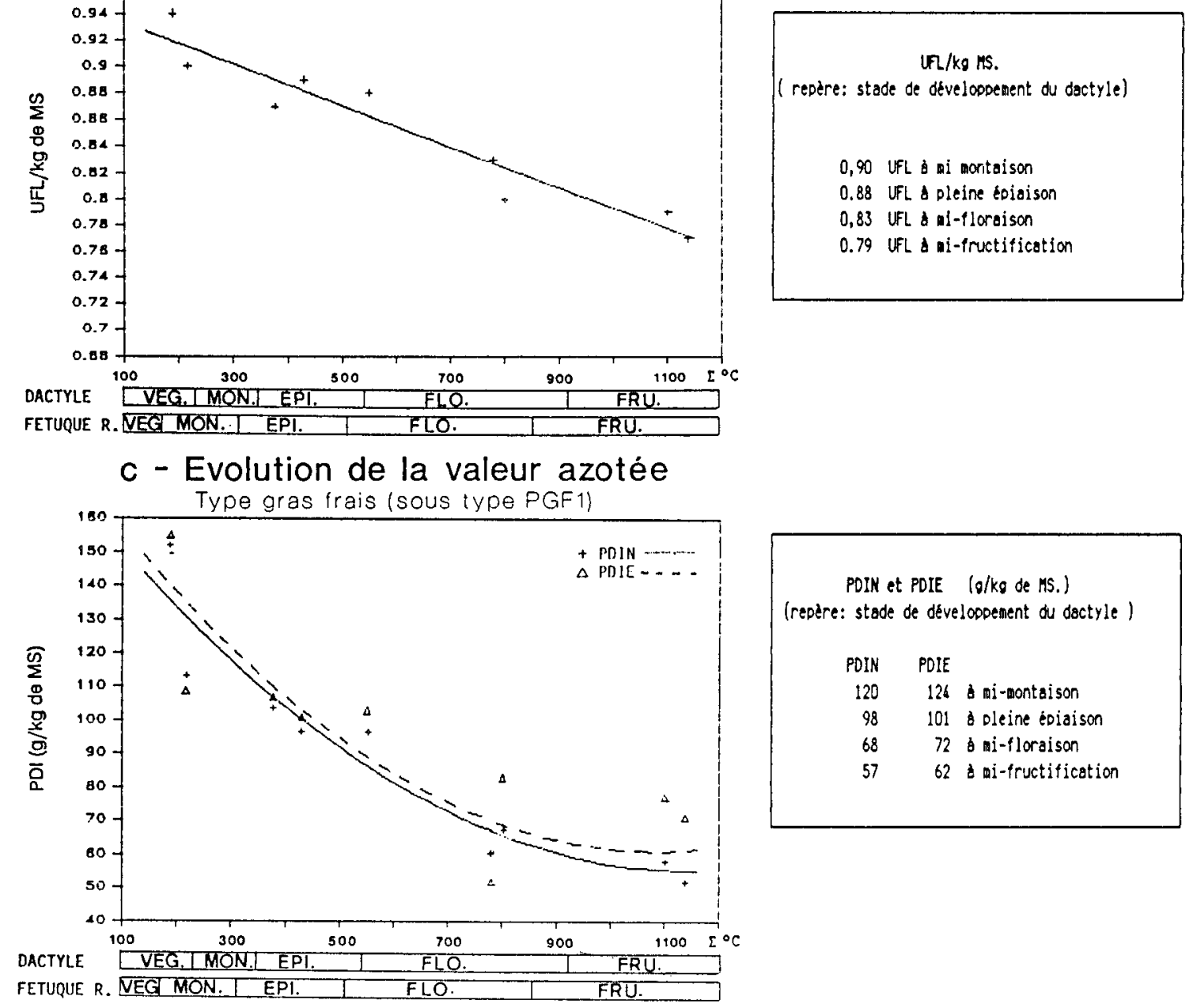

Fig 6. Dynamique de croissance et évolution de la valeur nutritive des pelouses grasses fraiches.

rubra, prises comme espèces-repères; au stade pleine épiaison, on atteint $70-80 \%$ de la production maximale produite; enfin c'est au stade de mi-floraison des graminées tardives citées que le pic de production de matière sèche du premier cycle est atteint. 
Ce rythme de croissance illustre le phénomène bien connu en montagne de "l'explosion de la végétation» au début de l'été, phénomène plus rapide que celui observé en plaine au printemps. Sur les alpages, le déneigement est généralement étalé en fonction des différences d'altitude, d'exposition, de pente et de topographie, ce qui aide l'alpagiste à mieux suivre le développement soudain de la végétation.

\section{Valeur nutritive}

L'évolution de la valeur nutritive énergétique (UFL $/ \mathrm{kg} \mathrm{MS}$ ) et de la valeur azotée ( $P D I N$ et $P D I E$ en $\mathrm{g} / \mathrm{kg} \mathrm{MS}$ ) au cours de la saison a été ajustée en fonction de la somme de degrésjours, de la même façon que pour la matière sèche. Les équations sont données en annexe 1. Pour tous les types de végétation, la chute de la valeur azotée est beaucoup plus rapide que celle de la valeur énergétique; elle est représentée, à titre d'exemple, sur les figures $6 \mathrm{~b}$ et $6 \mathrm{c}$, pour les pelouses grasses fraîches.

\section{Modèle de répartition des types de végétation sur un alpage}

Il est non seulement important de reconnaître les différents types de végétation sur une unité, mais aussi de bien les percevoir dans l'espace et d'en maîtriser les règles de répartition, en fonction des conditions d'utilisation et de milieu : leur connaissance éclairera en effet les choix à opérer en matière d'amélioration et d'aménagement de l'alpage.

Sur un alpage donné, où le système pastoral et les conditions lithologiques peuvent être considérées en première approche comme homogènes, les liaisons principales à prendre en compte pour analyser la répartition de la végétation et des ressources pastorales correspondantes sont à considérer à 2 niveaux : le niveau alpage et le niveau versant.

\section{Le niveau alpage, ou quartier d'alpage}

Les différences s'organisent en fonction des pratiques de pâturage et de restitutions (fig $7 \mathrm{a}$ ). $\mathrm{Ce}$ sous-modèle général propose quatre zones en fonction des pratiques des alpages laitiers.

\section{Zone I et II : influence prédominante des pratiques pastorales}

Zone I: zone située en périphérie immédiate des lieux de repos nocturne où les restitutions sont supérieures aux prélèvements par le pâturage, pouvant aller jusqu'à l'excès, ce qui entraîne le développement de pelouses grasses nitrophiles.
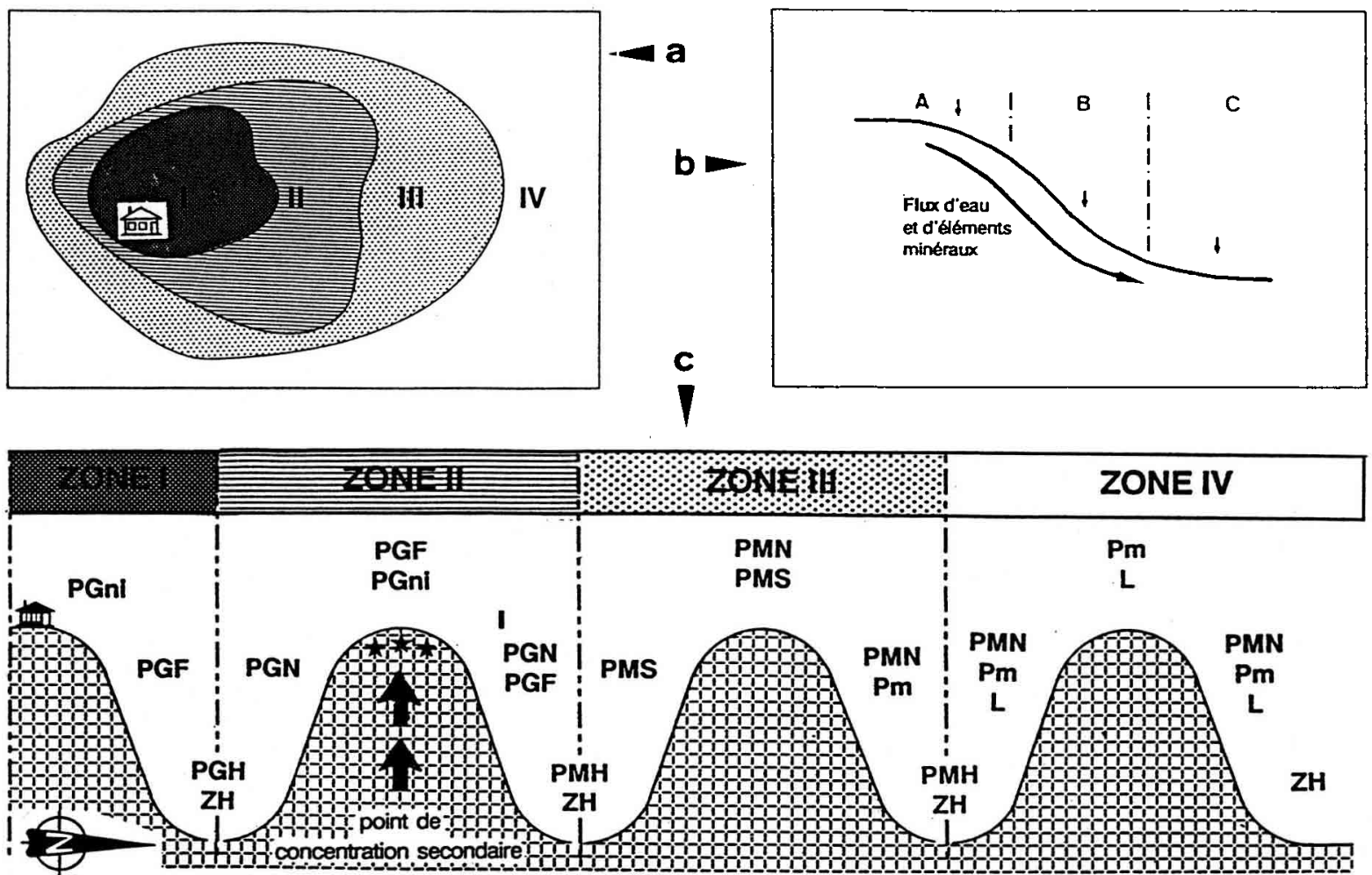

Fig 7. Modèle général de répartition spatiale des types de végétation sur un alpage laitier : en fonction des pratiques pastorales (a); au niveau d'un versant (b); influence combinée des pratiques pastorales et de la position topographique (c). 
Zone II : zone située en périphérie des précédentes ou autour des pôles d'attraction secondaire du troupeau (lieux de repos diurne, d'abreuvement, de pachonnée occasionnelle...); sur ces zones, les restitutions et le pâturage sont importants, en proportions relativement équilibrées. C'est le domaine des pelouses grasses où s'expriment les nuances liées au milieu (stations humides, fraîches, ou modérément sèches).

La concentration des restitutions dans ces deux premières zones représente un transfert interne de fertilité au niveau de l'alpage, au détriment des autres zones.

\section{Zone III : influence équilibrée \\ des pratiques pastorales \\ et des conditions de milieu}

II s'agit d'une zone encore plus éloignée des lieux de concentration du troupeau, où l'activité de pâturage reste importante, mais où les prélèvements l'emportent sur les restitutions. L'action du milieu vient compenser ou renforcer ce déséquilibre par endroits; il en résulte une mosaïque de végétations allant de faciès très appauvris, pelouses maigres acidophiles, à des pelouses de qualité moyenne.

\section{Zone IV : influence prépondérante des facteurs du milieu}

Il s'agit de la zone la plus éloignée de l'alpage (bordure, ou difficile d'accès) : un pâturage trop faible ou irrégulier et des restitutions négligeables laissent s'installer des formations peu intéressantes d'un point de vue pastoral (pelouses maigres, landes) à des degrés et à des vitesses dépendant des autres facteurs du milieu.

\section{Le niveau versant, ou unité géomorphologique}

Les facteurs du milieu interviennent fortement : à l'altitude et à l'exposition qui sont des facteurs majeurs en montagne, s'ajoutent les apports d'eau et d'éléments fertilisants pour lesquels la position topographique fournit un bon indicateur synthétique (Van Oort et Dorioz, 1982). Les cas de figure peuvent être très variés en fonction de l'allure générale du versant, des possibilités d'alimentation en amont (falaise calcaire) ou de drainage en aval, du pendage et de la nature des matériaux lithologiques et pédologiques (Dorioz et Trevisan, 1989 et 1990).
Nous prendrons comme exemple celui d'un versant régulier, isolé et établi sur matériaux carbonatés, avec un pendage parallèle au sol. Dans le contexte climatique des Alpes du Nord, en raison d'une pluviométrie élevée, les différences de végétation s'établissent comme suit le long du profil (fig $7 b$ ) :

\section{Zone A : haut de versant}

En l'absence de pratiques pastorales compensatrices, les contraintes du milieu favorisent l'installation de végétations susceptibles d'exprimer des déficits en eau et en substances nutritives, de types pelouses moyennes sèches ou maigres acidophiles.

\section{Zone B : mi-versant}

Les flux réguliers d'eau et de matière par lesquels les apports compensent les départs favorisent l'émergence de types de végétation dont l'ambiance est fraîche à modérément sèche (= neutre). Selon les conditions de milieu ou d'utilisation, une évolution est possible des pelouses grasses aux pelouses maigres.

\section{Zone $C$ : bas de versant}

L'accumulation d'eau et de matière favorise l'émergence des pelouses grasses ou, en cas d'excès d'eau, des zones humides.

En conclusion, le modèle général proposé intègre ces deux niveaux : il découle directement de la superposition des deux sousmodèles précédents, en fonction des pratiques et du milieu. Un alpage peut ainsi se schématiser comme une suite de toposéquences élémentaires (versants) dans chacune des quatre grandes zones d'influence des conditions d'utilisation (fig 7c).

D'une façon générale, la probabilité de rencontrer plusieurs types en un même point de l'alpage va croissant au fur et à mesure que l'on s'éloigne du chalet : la diversité des facteurs du milieu s'y exprime d'autant mieux en l'absence du pâturage régulier, et plus encore des restitutions directes ou par épandage. Néanmoins, lorsque les conditions de milieu sont particulièrement marquées, on peut plus exceptionnellement, même en proximité des chalets (zone l et II), rencontrer des types plus caractéristiques du milieu que de l'effet des pratiques. C'est le cas notamment des pelouses nivales associées aux stations à long enneigement, ou des zones humides, particulièrement favorables à l'engorge- 
ment. Les premières ne sont pas mentionnées sur la figure $7 \mathrm{c}$.

\section{Hypothèses d'évolution de la végétation des alpages laitiers}

D'une manière générale, il est vraisemblable qu'en cas d'abandon des pratiques traditionnelles de fertilisation organique, en raison notamment de la réduction de main d'œuvre, les pelouses grasses évolueront vers des pelouses de type moyen, tandis qu'à l'inverse une redistribution des restitutions animales est susceptible de faire évoluer ces dernières vers des pelouses de type gras. Cette évolution est cependant plus facile à envisager pour les types qualifiés de frais ou neutres, où les conditions de milieu ne présentent pas de facteur limitant trop accusé. L'évolution est certainement plus complexe pour les milieux humides où la gestion des excès d'eau jouera certainement un rôle primordial, et plus encore pour les pelouses moyennes sèches dont l'amélioration ne pourra être que probablement très lente ou très limitée, en l'absence d'irrigation, ou mieux d'irrigation fertilisante avec le lisier.

En ce qui concerne, les pelouses maigres acidophiles, les contraintes naturelles de ces milieux (degré d'acidification des sols, courte durée de végétation) font que ces pelouses évoluent assez lentement. Néanmoins, une diminution des charges animales ou un abandon du pâturage entraînerait une évolution des pelouses à Nardus stricta, vers des landes à myrtilles et, à terme, vers des landes à rhododendron; la vitesse et l'ampleur de ces phénomènes restent à préciser.

L'évolution des pelouses maigres acidophiles vers des pelouses d'intérêt pastoral plus grand nécessiterait une rénovation complète du mode d'exploitation de ces milieux. L'amélioration se discutera sur examen de la composition du peuplement : en l'absence de bonnes plantes fourragères (légumineuses ou bonnes graminées essentiellement) qui auraient pu s'exprimer sous l'effet conjugué d'une fumure minérale et d'un pâturage équilibré, les possibilités d'amélioration resteront vraisemblablement limitées (Dietl et al, 1981).

Pour les autres types de végétation, les possibilités d'évolution paraissent beaucoup plus limitées en raison de la sévérité des contraintes du milieu :
- le long enneigement des pelouses nivales bloque plus ou moins l'évolution de ces milieux;

- le degré d'acidification des sols des landes impose une vitesse d'évolution lente; toutefois, la diminution des charges animales ou l'abandon complet du pâturage ne peuvent qu'entraîner le développement des ligneux présents et transformer les landes ouvertes en landes fermées (taux de recouvrement des ligneux $>50 \%$ ) qui n'ont plus ici aucun intérêt pastoral;

- la durée annuelle de l'engorgement d'eau des zones humides règle leurs possibilités d'évolution; si le drainage n'est plus assuré, il semblerait que l'on passe successivement de l'une à l'autre des variantes décrites plus haut pour arriver au stade irréversible des marécages, qui devraient être conservés en l'état pour leur rôle de zone tampon (régulation hydrique, dénitrification, désulfuration).

\section{CONCLUSION}

Cette typologie de la végétation des alpages à vaches laitières des Alpes du Nord présente un double intérêt : c'est un véritable outil de gestion pour le développement, et c'est une étape déterminante pour la poursuite d'autres travaux de recherche sur la dynamique de la végétation.

La classification de l'ensemble des végétations en un nombre limité de types (11 principaux et 25 sous-types) demeure suffisamment précise pour rendre compte de la diversité existante pour des applications pastorales; de plus les critères de caractérisation retenus facilitent la reconnaissance de la végétation. Une clé de détermination des types de végétation a été élaborée, leur différenciation est basée sur l'appréciation conjointe de critères morphologiques (hauteur d'herbe), de la nature des espèces dominantes et indicatrices (25 pour la typologie simplifiée, une cinquantaine pour la typologie détaillée) et des conditions de milieu et d'utilisation du secteur étudié (Bornard et al, 1991).

Par ailleurs, grâce aux caractéristiques associées à chaque type, la typologie devient un outil de diagnostic pastoral, qui permet d'apprécier les différentes valeurs d'usage d'une végétation : productivité pondérale, valeur nutritive, charge animale, et d'apporter les principales recommandations d'utilisation pour l'entretien ou l'amélioration de ces végétations. Cette fonction a été concrétisée par l'élaboration de fiches techniques à l'usage des techniciens agricoles. Elles 
ont été élaborées par un groupe de travail associant chercheurs et techniciens du développement (Bornard et al, 1991).

Pour faciliter la reconnaissance et la localisation des types de végétation à un niveau opérationnel par les agents de terrain, il est possible d'effectuer un prézonage cartographique sur des alpages par utilisation d'images satellitaires; ces dernières permettent aussi d'envisager la réalisation d'inventaires des grandes catégories de couvert végétal, au niveau régional. Compte tenu de la nature des informations radiométriques et de la résolution spatiale fournie par les capteurs, il est nécessaire de disposer au préalable de typologies simplifiées de la végétation à valeur régionale (Bernard-Brunet et al, 1989). Le présent travail peut être la base de cette nouvelle approche cartographique.

Au cours de la période récente, des transformations profondes atteignent les alpages lai- tiers: une nette régression des disponibilités en main d'œuvre a conduit à l'abandon de certaines pratiques traditionnelles qui contribuaient à l'entretien du pâturage; à l'inverse, une augmentation de la productivité des animaux incite certains alpagistes à augmenter les ressources pastorales par la mise en œuvre de nouvelles techniques plus intensives. Face à ces profondes mutations, il est important d'accroître nos connais-sances sur l'évolution de la végétation de ces pâturages d'altitude, afin d'en préciser les trajectoires (Balent, 1987), la vitesse et les facteurs d'évolution. L'élaboration de la typologie a constitué une étape déterminante pour la poursuite du travail. Cette phase a permis de sélectionner les types de végétation à étudier, et de bâtir les premières hypothèses d'évolution en mettant en évidence les paramètres du milieu naturel et des pratiques pastorales, facteurs d'évolution dont on cherche à définir le rôle.

Annexe 1. Ajustement de la production de matière sèche et de la valeur nutritive, en fonction des sommes de températures comptées depuis le déneigement.

\begin{tabular}{|c|c|c|c|c|c|c|}
\hline \multirow{2}{*}{$\begin{array}{l}\text { Type de } \\
\text { pelouse }\end{array}$} & \multicolumn{2}{|c|}{ Variable expliquée } & \multirow[t]{2}{*}{ Équations } & \multirow[t]{2}{*}{$\mathrm{N}$} & \multicolumn{2}{|c|}{ Précision } \\
\hline & & & & & SR & $\mathrm{R}$ \\
\hline Grasse & MS & (tha) & $-6,48 \mathrm{E}-6 \times \mathrm{TTEMP}^{2}+0,0107 \times \mathrm{TEMP}-1,17$ & 18 & 0,249 & 0,98 \\
\hline Fraîche & UFL & (UFL/kg MS) & $-1,55 \mathrm{E}-4 \times \mathrm{TEMP}+0,948$ & 9 & 0,018 & $-0,96$ \\
\hline$\left(\mathrm{PGF}_{1}\right)$ & PDIN & $(\mathrm{g} / \mathrm{kg} \mathrm{MS})$ & 8,70 E-5 $\times$ TEMP $^{2}-0,200 \times$ TEMP +170 & 9 & 11,7 & 0,95 \\
\hline Grasse & MS & (t/ha) & $-5,87 \mathrm{E}-6 \times$ TEMP $^{2}+8,88 \mathrm{E}-3-1,17$ & 10 & 0,316 & 0,85 \\
\hline Neutre & UFL & (UFL/kg MS) & $-2,14 \mathrm{E}-4 \times \mathrm{TEMP}+0,975$ & 5 & 0,019 & $-0,98$ \\
\hline (PGN) & PDIN & (g/kg MS) & 4,9 E- $5 \times$ TEMP $^{2}-0,129 \times$ TEMP +135 & 5 & 8,47 & 0,98 \\
\hline Grasse & MS & (t/ha) & $-5,12 \mathrm{E}-6 \times \mathrm{TEMP} 2+8,44 \mathrm{E}-3 \times \mathrm{TEMP}-0,778$ & 27 & 0,571 & 0,75 \\
\hline Humide & UFL & (UFL/kg MS) & $-2,99 E-4 \times$ TEMP $+0,987$ & 9 & 0,0463 & $-0,87$ \\
\hline$(\mathrm{PGH})$ & PDIN & $(\mathrm{g} / \mathrm{kg} \mathrm{MS})$ & 3,1 E-5 $\times$ TEMP $2-0,131 \times$ TEMP + 159 & 9 & 12,7 & 0,91 \\
\hline Moyenne & & & & & & \\
\hline $\begin{array}{l}\text { Neutre } \\
\text { (PMN) }\end{array}$ & MS & (t/ha) & $-1,03 E-5 \times$ TEMP $^{2}+0,0139 \times$ TEMP $-2,90$ & 13 & 0,41 & 0,80 \\
\hline Moyenne & & & & & & \\
\hline $\begin{array}{l}\text { Humide } \\
\left(\mathrm{PMH}_{3}\right)\end{array}$ & MS & (t/ha) & $-3,58 \mathrm{E}-6 \times \mathrm{TEMP}^{2}+5,49 \mathrm{E}-3 \times \mathrm{TEMP}+0,294$ & 4 & 0,304 & 0,93 \\
\hline Pré & MS & (t/ha) & $-2,88 \mathrm{E}-6 \times \mathrm{TEMP}^{2}+4,61 \mathrm{E}-3 \times \mathrm{TEMP}-0,417$ & 18 & 0,118 & 0,94 \\
\hline Nivale & UFL & (UFL/kg MS) & $-2,36 \mathrm{E}-4 \times \mathrm{TEMP}+0,998$ & 5 & 0,014 & $-0,97$ \\
\hline$\left(\mathrm{PN}_{3}\right)$ & PDIN & $(g / k g$ MS) & 1,58E-4 x TEMP $2-0,281 \times$ TEMP + 195 & 5 & 4,72 & 0,99 \\
\hline
\end{tabular}

TEMP : somme des températures en ${ }^{\circ} \mathrm{C}$, supérieures à $0^{\circ} \mathrm{C}$, comptées depuis le déneigement de la station. E- $n=10^{-n}$; exemple : $-6,48 \mathrm{E}-6=-0,00000648$. 


\section{REMERCIEMENTS}

Ce travail a bénéficié de la collaboration technique de $C$ Bernard-Brunet (CEMAGREF) et des conseils de nos collègues $J$ Bernard-Brunet et $P$ Cozic. Nous remercions les stagiaires du CEMAGREF qui ont largement contribué au recueil des données et à leurs premières analyses : $B$ Blanchet, $F$ Bourchanin, A Escudero, L Haltel, I Philippot. Ces études ont été réalisées dans le cadre du programme de recherchedéveloppement sur l'agriculture de montagne du «GIS Alpes du Nord". Ce travail a été présenté à la 7 e réunion du sous-réseau FAO des herbages de montagne, Nyon (Changins) Suisse 5-8, septembre 1991.

\section{RÉFÉRENCES}

Balent G (1987) Structure, fonctionnement et évolution d'un système pastoral. Le pâturage vu comme un facteur écologique piloté dans les Pyrénées centrales. Thèse univ Rennes, $146 \mathrm{p}$

Bernard-Brunet J, Cozic P (1986) Prise en compte de la diversité spécifique dans l'analyse et la modélisation des effets du climat sur la croissance de I'herbe dans les étages alpin et subalpin. Agrométérologie des régions de moyenne montagne, Toulouse 16-17 avril 1986. Colloq INRA 39, 407-430

Bernard-Brunet J, Dubost M, Bornard A (1989) Utilisation de la télédétection spatiale pour la discrimination des surfaces pastorales d'altitude. Bull Soc Fr Photogram Télédétect 115, 59-61

Bornard A, Blanchet B, Brau-Nogué C, Folliet X, Larrieu G, Hauwuy A, Trévisan D (1991) Typologie de la végétation des alpages des Alpes du Nord. Fiches techniques. GIS Alpes du Nord, Chambéry, $40 \mathrm{p}$

Cozic P (1987) Une méthode de diagnostic pastoral, de la composition de la végétation à la charge animale à préconiser. In : Exploitation de pelouses et landes subalpines par des bovins et des ovins. CEMAGREF-INERM, Grenoble, 211, 171-197

Daget P, Poissonnet J (1971) Une méthode d'analyse phytologique des prairies. Critères d'application. Ann Agron 22 (1), 5-41

Delpech R (1970) Contribution à l'étude de quelques prairies et alpages du massif de la Vanoise. Trav Sci Parc Natl Vanoise I, 39-74

Delpech R (1979) Réflexions sur quelques problèmes biologiques soulevés par l'exploitation pastorale en montagne. In : Utilisation par les ruminants des pâturages d'altitude et parcours méditerranéens. INRA, Versailles, 45-56

Dietl W, Berger P, Ofner M (1981) Die Kartierung des Pflanzenstandortes und der futterbaulichen Nutzungseignung von Naturwiesen. FAP, AGFF Zurich-Reckenholz, $45 \mathrm{p}$

Dorée A (1976) Évaluation des potentialités fourragères en montagne; application aux zones de moyenne altitude. CEMAGREF-INERM, Grenoble, $94,127 \mathrm{p}$

Dorioz JM, Party JP (1987) Dynamique écologique et typologique de territoires pastoraux des Alpes du Nord. I. Analyse de l'organisation agroécologique d'un alpage de référence. Acta Oecol Oecol App/8, 257-280

Dorioz JM (1987) Dynamique écologique et typologique de territoires pastoraux des Alpes du Nord. II. Analyse des facteurs de la valeur pastorale dans un secteur de référence. Conséquences pour une typologie régionale des territoires pastoraux. Acta Oecol Oecol App/ 8, 283-300

Dorioz JM, Trevisan D (1989) Couverture pédologique et surfaces fourragères de montagne. I. Éléments pour un diagnostic agronomique. INRA, Thonon, doc ronéoté, GIS Alpes du Nord, $63 \mathrm{p}$

Dorioz JM, Trevisan D (1990) Couverture pédologique et surfaces fourragères de montagne. II. Les relations sols-paysages. INRA, Thonon, doc ronéoté, GIS Alpes du Nord, $45 p$

Dorioz JM, Van Oort F (1991) Approche agropédologique des zones pastorales sur calcschistes sédimentaires (Beaufortain-Alpes du Nord). II. Répartition des sols et des valeurs d'usage pastoral. Agronomie 11, 395-409

Dubost M, Jouglet JP (1983) La végétation des alpages et sa productivité fourragère, approche phyto-écologique. In : Pastoralisme montagnard, recherches en Briançonnais. CEMAGREF-INERM, Grenoble, 15-62

Dubost M, Bornard A (1985) Essai de typologie à usage pastoral des grands groupements végétaux des alpages laitiers des Alpes du Nord. CEMAGREFINERM, Grenoble, doc ronéoté, $11 \mathrm{p}$

Dubost M, Bornard A (1987) Pratiques pastorales des alpages laitiers dans les Alpes françaises du Nord. Communication $5 e$ réunion sous-réseau des herbages de montagnes-FAO. Bled (Yug), $33 \mathrm{p}$

Jouglet JP, Jacquier C (1976) Recherches sur les écosystèmes montagnards : méthode d'analyse globale et quantitative. Application : productivité des pâturages de haute-altitude. CEMAGREF-INERM, Grenoble, 98, $405 \mathrm{p}$

Jouglet JP, Bernard-Brunet J, Dubost M (1982) Phénologie de quelques espèces des pelouses subalpines et alpines du Briançonnais. Fourrages 92, 67-90

Lacoste A, Roux M (1971) L'analyse multidimentionnelle en phytosociologie et en écologie. Application à des données de l'étage subalpin. I. L'analyse des données floristiques. Acta Oecol Oecol Plant 7, 125-146

Lambertin M (1987) Les écosystèmes d'altitude et le pâturage ovin, éléments pour la gestion d'un alpage. Thèse univ, Montpellier, $200 \mathrm{p}$

Landolt E (1977) Ökologische Zeigerwerte zur Schweizer Flora. Veroffentlichungen des Geobotanischen Institutes der ETH, Stiftung Rubel, Zurich, $208 p$

Legros JP (1986) Cartographie des paysages pédologiques dans les Alpes humides. Exemple du Chablais. Colloq INRA 39, 119-127 
Legros JP, Party JP, Dorioz JM (1987) Répartition des milieux calcaires, calciques et acidifiés en haute montagne calcaire humide; conséquences agronomiques et écologiques. Doc Cartogr Ecol vol XXX, 137-157

de Montard F, Gachon L (1978) Contribution à l'étude de l'écologie et de la productivité des pâturages d'altitude des Monts Dore. I. Application de l'analyse factorielle des correspondances à l'analyse de la végétation. Ann Agron 29 (3), 277-310

Ozenda P (1985) La végétation de la chaine alpine dans l'espace montagnard Européen. Masson, Paris, $330 p$

Philippe T (1982) Contribution à l'étude des pelouses subalpines cartusiennes (alpage de la Grande Sure en Charteuse) Thèse, univ Grenoble, $168 \mathrm{p}$

Plantureux S, Bonischot R, Guckert A (1987) Effet des techniques d'intensification sur l'évolution de la végétation de prairies permanentes lorraines. Acta Oecol Oecol Appl 8 (3), 229-246
Poissonnet P, Poissonnet J (1969) Étude comparée de diverses méthodes d'analyse de la végétation des formations herbacées denses et permanentes. Conséquences pour des applications agronomiques. CNRS Montpellier doc $\mathrm{N}^{\circ} 50,120 \mathrm{p}$

Richard L, Pautou G (1982) Carte de la végétation au 200 000e; Alpes du Nord et Jura méridional. CNRS, Paris, $316 p$

Van Oort F, Dorioz JM (1982) Les sols sous prairie et alpage du Beaufortain. INRA, Sci Sol, Versailles, $47 \mathrm{p}$

Van Oort F, Dorioz JM (1991) Approche agropédologique des zones pastorales sur calcschistes sédimentaires (Beaufortain-Alpes du Nord). I. Caractères de l'altération, propriétés hydriques et porales associées. Agronomie 11, 293-303

Vertes F (1983) Contribution à l'étude phytosociologique et écologique des prairies et alpages de moyenne Tarentaise. Application à l'évaluation des potentialités de la vallée de Pesey Nancroix. Thèse INAPG, Paris $160 p$ 